\title{
Regge Poles in High-Energy Electron Scattering*
}

\author{
R. Blankenbecler, L. F. CoOK, Jr., and M. L. Goldberger \\ Palmer Physical Laboratory, Princeton University, Princeton, New Jersey
}

(Received July 19, 1962)

\begin{abstract}
The possibility that the photon is described by a Regge trajectory is considered, and the effect of this assumption on the analysis of electron-pion, electron-nucleon, and electron-helium scattering is examined in some detail. Partial-wave projections for the various amplitudes are made in the annihilation channel, and a multiparticle unitarity condition is formally imposed by use of the $N / D$ matrix formulation. Since the photon does not have a fixed spin of one, the spin matrix structure is considerably more complicated than in the conventional theory. The amplitudes are written in terms of the Regge poles corresponding to the photon, $\rho-\omega$ meson, etc., and the resulting cross sections are given in the interesting high-energy limit. In contrast to the usual analysis, where form factors depend only on the momentum transfer, we find a larger number of independent functions which depend on the energy as well, however, in a characteristic manner. That is, the essential change due to the Regge behavior of the photon is an over-all nonintegral power of the energy occurring in the cross section. The effect of this factor can be experimentally tested and this possibility is discussed.
\end{abstract}

\section{A. INTRODUCTION}

$T$ HE idea that all strongly interacting particles are nonelementary in the technical sense of not being associated with a quantized wave field is a very attractive one. In fact, the possibility of associating such particles with trajectories of so-called Regge poles of the $S$ matrix regarded as an analytic function of a complex angular momentum has been widely discussed..$^{1-4}$ Unfortunately, it has not yet been possible to establish theoretically any of the "desired" properties of Regge trajectories in a relativistic theory. In any case, the trajectory concept seems to be a very useful one for high-energy physics phenomenology, ${ }^{5}$ and the question of "elementarity" can be tested experimentally."

It is somewhat disturbing that any theory based on such general principles as unitarity and analyticity should single out, as has been conjectured by Chew and Frautschi, ${ }^{2}$ only the strong interactions. The photon would seem to be the most important missing particle. ${ }^{6}$ One could attempt to avoid including the photon in the analysis by mentioning the "weakness" of the electromagnetic coupling, but as we shall see, if the Regge hypothesis is true in strong interactions, then there are kinematical regimes in which the "weak" electromagnetic effects dominate the strong interactions.

We would like to direct attention to the possibility ${ }^{7}$ that the photon is not an elementary particle but that

\footnotetext{
* Supported in part by the Air Force Office of Scientific Research, Air Research and Development Command.

${ }^{1}$ R. Blankenbecler and M. L. Goldberger, International Conference on Weak and Strong Interactions at LaJolla, June 1961 (unpublished), and Phys. Rev. 126, 766 (1962).

${ }_{2}^{2}$ G. F. Chew and S. Frautschi, Phys. Rev. Letters 7, 394 (1961).

${ }^{3}$ V. Gribov, J. Exptl. Theoret. Phys. (U.S.S.R.) 41, 667,1962

(1961) [translation: Soviet Phys.-JETP 14, 478, 1395 (1962)].

${ }^{4}$ S. Frautschi, M. Gell-Mann, and F. Zachariasen, Phys. Rev. 126, 2204 (1962).

5 B. Udgaonkar, Phys. Rev. Letters 8, 1942 (1962) and B. Udgaonkar and M. Gell-Mann, ibid. 8, 347 (1962).

${ }^{6}$ This possibility was first mentioned in reference 4.

${ }^{7}$ R. Blankenbecler, L. F. Cook, and M. L. Goldberger, Phys. Rev. Letters 8, 463 (1962). This possibility has also been discussed by P. Freund, M. Gell-Mann, J. Sakarai, and F. Zachariasen (unpublished).
}

it, too, is a Regge trajectory, with perhaps such a small slope that for most purposes it behaves as a massless, spin one object, properly described by the conventional formalism. It seems to us that in much the same way as one proves the necessity for quantizing the electromagnetic field by considering its interaction with quantized matter, it should be possible to prove that if all charged particles are merely Regge trajectories, then the photon must be one also. However, we have not been able to construct a convincing version of this argument, nor have we been able to formulate a Regge theory of electrodynamics incorporating gauge invariance, etc. Instead, we have worked in a more phenomenological fashion to see if there are observable consequences of such a hypothesis in the regime of large energy and momentum transfer phenomena involving charged particles. We have concentrated on electron scattering experiments because they seem to offer the cleanest experimental possibilities.

To illustrate the fact that there can be no real distinction between the weak interactions and the strong interactions if the Regge hypothesis is correct for the latter alone, let us consider proton-proton scattering. If we assume that the matrix element is dominated by the vacuum trajectory contribution at high energies, we find (for one of the matrix invariants)

$$
M_{R}=-\left[\frac{1+e^{-i \pi \alpha(t)}}{\sin \pi \alpha(t)}\right] \alpha(t) \frac{G^{2}(t)}{\mu^{2}} \gamma^{(1)} \cdot \gamma^{(2)} z^{\alpha(t)-1}
$$

where

$$
z=2\left[2 M E+\frac{1}{2} t\right] /\left(t-4 M^{2}\right),
$$

$E$ is the laboratory energy, and $t$ is the usual invariant squared momentum transfer. The spinors associated with the $\gamma$ matrices have been omitted and $G(t)$ is a form factor $[G(0)=1]$. The vacuum Regge trajectory is $\alpha(t)[\alpha(0)=1]$ and the factors have been chosen to yield a total cross section of $40 \mathrm{mb}$.

On the other hand, if the photon were elementary, we would have from the one-photon, exchange graph; 
with $F(t)$ the usual form factor,

$$
M_{P}=-\left(e^{2} / t\right) F^{2}(t) \gamma^{(1)} \cdot \gamma^{(2)} .
$$

In this expression, $e^{2} / 4 \pi=1 / 137$, and it is expected that $G \simeq F$. We see that the "weak" interaction overtakes the "strong" when $z$ is sufficiently large. For example, if $t \simeq-50 \mu^{2}$ where $\alpha \simeq 0,{ }^{4}$ the photon is more important than the vacuum trajectory for lab energies larger than $430 \mathrm{BeV}$. If the momentum transfer is increased to $t \simeq-100 \mu^{2}$, where $\alpha \simeq-1$, then the required lab energy is reduced to $50 \mathrm{BeV}$ for equality. Even at $30 \mathrm{BeV}$ the electromagnetic corrections are quite significant, of the order of $20 \%$ in the cross section for $t \simeq-50 \mu^{2}$.

The natural and obvious way of avoiding this unreasonable dominance of an "elementary" photon is to assume that it too is described by a Regge trajectory. ${ }^{7}$ We feel that this would provide a dramatic unification in the present understanding of weak and strong interactions. Whether the very weak beta-decay interactions should also be unified is a point that we hope to return to later. If this is the case with the photon, then one finds an energy dependence in form factors which in conventional quantum electrodynamics depend only on the momentum transfer. Explicit experimental tests to explore the possible Regge character of the photon will be described in the last section after treatment of electron-pion, electron-nucleon (isovector and isoscalar), and electron-alpha particle scattering. The most obvious difference between the conventional analysis and the Regge analysis carried out here is the fact that all invariant amplitudes contribute to the matrix element. For example, in electron-pion scattering there are two independent functions of energy and momentum transfer instead of the usual single form factor depending only on $t$. Similarly, in electron-nucleon scattering there are six functions in place of two form factors. We are well aware that the two-photon exchange graphs in the ordinary theory give rise to such a complication and we will return to this important point later.

All of the analysis in Sec. B is applicable to many other reactions in addition to electron scattering. In particular, the solution of the coupled channel problem within the Regge framework should find wide application. The isotopic vector pion and nucleon form factors will be discussed as a coupled system by use of the matrix $N / D$ formulation. ${ }^{8}$ The isotopic scalar nucleon form factor will then be treated in an analogous manner. ${ }^{9}$ Then the alpha-particle form factor, which requires a discussion of anomalous thresholds in the Regge approach, will be discussed. Finally, we remark that the methods used here would allow an explicit attack on the detailed structure of the matrix element in terms of the $\pi-\pi$ resonance trajectory, the nucleon

${ }^{8}$ J. D. Bjorken, Phys. Rev. Letters 4, 473 (1960) and M. Nauenberg, thesis (unpublished).

${ }^{9}$ For a conventional approach see R. Blankenbecler and J. Tarski, Phys. Rev. 125, 782 (1962). trajectory, and the $(3,3)$ resonance trajectory. Since we are interested for the present only in the effect of the Regge hypothesis on the general structure of the matrix elements, this calculational possibility will not be utilized here.

\section{B. SPECIFIC REACTIONS}

In this section we wish to examine four specific processes, and in particular to study the structure of the matrix elements when it is assumed that the photon is represented as a Regge trajectory rather than an "elementary" particle. In general, our procedure will be as follows: We consider the partial-wave projection in the annibilation channel for the invariant amplitudes appropriate to the reaction in question and impose a two-body unitarity relation on the projected amplitudes. We formally solve for the amplitudes by means of the $N / D$ technique and obtain convenient expressions for the partial-wave amplitudes. These solutions are then formally extended to include inelastic contributions. Next, we write the partial-wave sum as a contour integral in the complex, angular-momentum plane, and we assume that the denominator function $D$ has zeros at complex values of the angular momentum as a function of the annihilation-channel energy. These zeros, or Regge poles, will be taken to represent the photon and other resonant states in the annihilation channel. Their effect on the cross section in the scattering channel will be obtained in the high-energy limit and compared to the perturbation theory result.

\section{Electron-Pion Scattering}

We consider $e-\pi$ scattering first, not only because it will be needed in the discussion of the processes that follow, but because it involves fewer algebraic complications than the other processes we consider involving electrons. It will thus serve as an example of our procedure, and we will give the analysis in some detail.

Consider the process in Fig. 1 in which two pions annihilate into an electron-position pair. Define the variables

$$
\begin{aligned}
s & =-\left(p_{1}-q_{1}\right)^{2}=m^{2}+1-\frac{1}{2} t+2 p q z_{t}, \\
t & =-\left(q_{1}+q_{2}\right)^{2}=4\left(p^{2}+m^{2}\right)=4\left(q^{2}+1\right), \\
u & =-\left(p_{1}-q_{2}\right)^{2}=m^{2}+1-\frac{1}{2} t-2 p q z_{t},
\end{aligned}
$$

where $p_{1}^{2}=p_{2}^{2}=-m^{2}, q_{1}^{2}=q_{2}^{2}=-1$, and $p$ and $q$ are the

Fig. 1. Definition of momenta for electron-pion scattering.

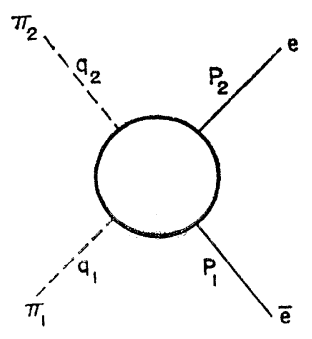


momenta of the electrons and pions, respectively, while

$$
z_{t}=\mathbf{p}_{1} \cdot \mathbf{q}_{1} / p q .
$$

All quantities are defined in the barycentric system of the annihilation channel. The matrix element, appropriate for the annihilation channel, can be written as

$$
f=\bar{u}\left(p_{2}\right)\left[a-\frac{1}{2} i \gamma \cdot\left(q_{1}-q_{2}\right) b\right] v\left(p_{1}\right),
$$

where the invariant amplitudes, $a$ and $b$, are functions of the variables $s, t, u$, alone. In first-order perturbation theory of quantum electrodynamics one easily sees that

$$
\begin{aligned}
& a=0, \\
& b=\frac{2 e^{2}}{t} \odot(t),
\end{aligned}
$$

where $P(t)$ is the pion form factor. We may define the helicity projections for $a$ and $b$, following Frazer and Fulco, ${ }^{10}$ by

$$
\begin{aligned}
a\left(t, z_{t}\right)+m \frac{q}{p} z_{t} b\left(t, z_{t}\right) & =-\frac{8 \pi}{p^{2}} \sum_{J}(2 J+1)(p q)^{J} f_{+}{ }^{J}(t) P_{J}\left(z_{t}\right) \\
b\left(t, z_{t}\right) & =-8 \pi \sum_{J}(2 J+1)(p q)^{J-1} f_{-}{ }^{J}(t) \frac{P_{J^{\prime}}\left(z_{t}\right)}{[J(J+1)]^{1 / 2}}
\end{aligned}
$$

where $P_{J}^{\prime}\left(z_{t}\right)$ means differentiation with respect to the argument of $P_{J}\left(z_{t}\right)$. It is convenient to separate $a$ and $b$ into even and odd parts where

and write Eq. (1.5) as

$$
\begin{aligned}
& a^{(e, o)}\left(t, z_{t}\right)= \pm a^{(e, o)}\left(t,-z_{t}\right), \\
& b^{(e, o)}\left(t, z_{t}\right)= \pm b^{(e, o)}\left(t,-z_{t}\right),
\end{aligned}
$$

$$
\begin{aligned}
a^{(e, o)}\left(t, z_{t}\right)+m-z_{t} b^{(o, e)}\left(t, z_{t}\right) & =-\frac{8 \pi}{p^{2}} \sum_{J}(2 J+1)(p q)^{J} f_{+}{ }^{J}(t) \frac{\left[P_{J}\left(+z_{t}\right) \pm P_{J}\left(-z_{t}\right)\right]}{2}, \\
p & \\
b^{(e, o)}\left(t, z_{t}\right) & =-8 \pi \sum_{J}(2 J+1)(p q)^{J-1} f_{-} J(t) \frac{\left[P_{J}^{\prime}\left(+z_{t}\right) \pm P_{J}{ }^{\prime}\left(-z_{t}\right)\right]}{2[J(J+1)]^{1 / 2}} .
\end{aligned}
$$

The Pauli principle then requires that $a^{(e)}$ and $b^{(o)}$ contribute to states of even isotopic spin, i.e., $I=0$ and 2 , while $a^{(o)}$ and $b^{(e)}$ contribute to states of odd isotopic spin, i.e., $I=1$.

In order to calculate the amplitudes in Eq. (1.6) we must obtain expressions for $f_{ \pm}^{J}(t)$. We accomplish this by requiring that a two-body unitarity condition be satisfied. In particular, we include only the two-pion state and have, therefore,

$$
\operatorname{Im} f\left(t, z_{t}\right)=\frac{q}{32 \pi^{2} t^{1 / 2}} \int d \Omega^{\prime} h^{*}\left(t, z^{\prime \prime}\right) f\left(t, z^{\prime}\right),
$$

where $d \Omega^{\prime}$ represents the angles of $q^{\prime}$ and $z^{\prime}, z^{\prime \prime}$ are

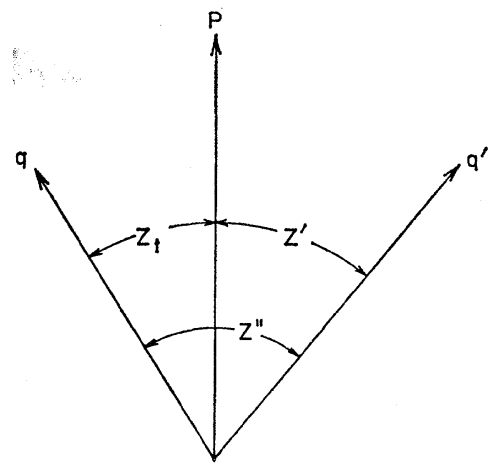

Fig. 2. Definition of angles in electronpion scattering. defined in Fig. 2. The amplitude $h\left(t, z^{\prime \prime}\right)$ is the $\boldsymbol{\pi}-\boldsymbol{\pi}$ scattering amplitude and satisfies the unitarity condition

$$
\operatorname{Im} h(t, z)=\frac{q}{32 \pi^{2} t^{1 / 2}} \int d \Omega^{\prime} h^{*}\left(t, z^{\prime \prime}\right) h\left(t, z^{\prime}\right) .
$$

The notation in Eq. (1.8) is obvious when compared with Eq. (1.7).

The unitarity condition in terms of $h^{J}(t)$ and $f_{ \pm}{ }^{J}(t)$ is easily obtained by using Eq. (1.6) together with

$$
h(t, z)=8 \pi \sum_{J}(2 J+1) q^{2 J} h^{J}(t) P_{J}(z) .
$$

We take Eq. (1.9) to represent the expansion for $I=1$, and we will confine our attention to this state in the remainder of this section. This, of course, means that only $a^{(o)}$ and $b^{(e)}$ are of interest to us. Equations (1.8) and (1.9) lead immediately to

$$
\operatorname{Im} h_{J}(t)=\frac{q^{2 J+1}}{t^{1 / 2}} h_{J}^{*}(t) h_{J}(t) \theta(t-4) .
$$

Equation (1.7) together with Eqs. (1.8) and (1.9) lead to a similar result for $f_{ \pm}{ }^{J}(t)$ and it is instructive to do this in some detail as it will be the general procedure in the following sections.

${ }^{10} \mathrm{~W}$. Frazer and J. Fulco, Phys. Rev. 117, 1609 (1960). 
We may rewrite Eq. (1.3) in two ways to simplify the evaluation of Eq. (1.7). In particular,

and

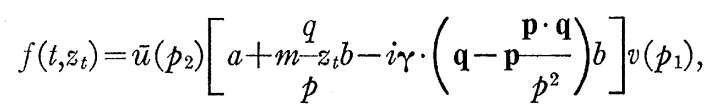

$$
\begin{aligned}
& f\left(t, z^{\prime}\right)=\bar{u}\left(p_{2}\right)\left[a-i \gamma \cdot \mathbf{q}^{\prime} b\right] v\left(p_{1}\right)
\end{aligned}
$$

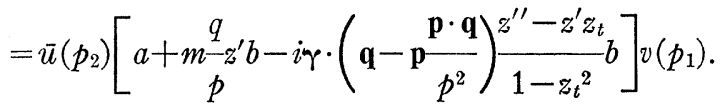

In both cases, one writes $\mathbf{q}$ or $\mathbf{q}^{\prime}$ in terms of an orthogonal system based on $\mathbf{p}$, e.g.,

$$
\mathbf{q}^{\prime}=\mathbf{p} \frac{\mathbf{p} \cdot \mathbf{q}^{\prime}}{p^{2}}+\left(\mathbf{q}-\mathbf{p} \frac{\mathbf{p} \cdot \mathbf{q}}{p^{2}}\right) \frac{\left[\mathbf{q}-\mathbf{p}\left(\mathbf{p} \cdot \mathbf{q} / p^{2}\right)\right] \cdot \mathbf{q}^{\prime}}{q^{2}-(\mathbf{p} \cdot \mathbf{q})^{2} / p^{2}}+(\mathbf{p} \times \mathbf{q}) \frac{(\mathbf{p} \times \mathbf{q}) \cdot \mathbf{q}^{\prime}}{p^{2} q^{2}-(\mathbf{p} \cdot \mathbf{q})^{2}} .
$$

In fact, the cross-product terms will not contribute to the amplitude as will be seen shortly.

We introduce Eqs. (1.11) and (1.12) with Eq. (1.9) into Eq. (1.7) and obtain

$$
\begin{aligned}
8 \pi \sum_{J}(2 J+1) \frac{(p q)^{J}}{p^{2}} & \operatorname{Im} f_{+}{ }^{J}(t) P_{J}\left(z_{t}\right)-8 \pi \sum_{J}(2 J+1)(p q)^{J-1} \operatorname{Im} f_{-} J \frac{P_{J}\left(z_{t}\right)}{[J(J+1)]^{1 / 2}} i \boldsymbol{\gamma} \cdot\left(\mathbf{q}-\mathbf{p} \frac{\mathbf{p} \cdot \mathbf{q}}{p^{2}}\right) \\
= & \frac{q(8 \pi)^{2}}{32 \pi^{2} t^{1 / 2}}\left\{\sum_{J, j}(2 J+1)(2 j+1) q^{2 J} \frac{(p q)^{j}}{p^{2}} h_{J}^{*}(t) f_{+}^{j}(t) \int d \Omega^{\prime} P_{J}\left(z^{\prime \prime}\right) P_{j}\left(z^{\prime}\right)\right. \\
& \left.\quad-i \boldsymbol{\gamma} \cdot\left(\mathbf{q}-\mathbf{p} \frac{\mathbf{p} \cdot \mathbf{q}}{p^{2}}\right) \sum_{J, j} \frac{(2 J+1)(2 j+1)}{[j(j+1)]^{1 / 2}} q^{2 J}(p q)^{j-1} h_{J}^{*}(t) f_{-}^{j}(t) \int d \Omega^{\prime} P_{J}\left(z^{\prime \prime}\right) P_{j}^{\prime}\left(z^{\prime}\right) \frac{z^{\prime \prime}-z^{\prime} z_{t}}{1-z_{t}{ }^{2}}\right\} .
\end{aligned}
$$

By use of the formulas

$$
\begin{array}{r}
\int d \Omega^{\prime} P_{J}\left(z^{\prime \prime}\right) P_{j}\left(z^{\prime}\right)=\frac{4 \pi}{2 J+1} \delta_{J j} P_{J}\left(z_{t}\right), \\
\int d \Omega^{\prime} P_{J}\left(z^{\prime \prime}\right) P_{j}^{\prime}\left(z^{\prime}\right) \frac{z^{\prime \prime}-z^{\prime} z_{t}}{1-z_{t}{ }^{2}}=\frac{4 \pi}{2 J+1} \delta_{J j} P_{J}\left(z_{t}\right),
\end{array}
$$

which fixes the normalization of the integrals we shall need, one can compare coefficients in Eq. (1.14) and obtain

$$
\operatorname{Im} f_{ \pm}^{J}(t)=\frac{q^{2 J+1}}{t^{1 / 2}} h_{J}^{*} f_{ \pm}^{J} \theta(t-4)
$$

If we assume the analytic structure of $h_{J}(t)$ is known, we can solve Eq. (1.16) to obtain an expression for $f_{ \pm}{ }^{J}$. Let us write, in the usual way,

$$
h_{J}(t)=N^{J}(t) / D^{J}(t),
$$

which satisfies Eq. (1.10) if $N^{J}$ is real for $t>4$ and

$$
D^{J}(t)=1-\frac{1}{\pi} \int_{4}^{\infty} d t^{\prime} \frac{\left[q\left(t^{\prime}\right)\right]^{2 J+1}}{\left(t^{\prime}\right)^{1 / 2}} \frac{N^{J}\left(t^{\prime}\right)}{t^{\prime}-t} .
$$

Thus, Eq. (1.16) is satisfied by

$$
f_{ \pm}^{J}(t)=r_{ \pm}^{J}(t) / D^{J}(t)
$$

where

$$
r_{ \pm}^{J}(t)=\frac{1}{\pi} \int_{-\infty}^{t_{0}} d t^{\prime} \frac{\operatorname{Im} f_{ \pm}{ }^{J}\left(t^{\prime}\right)}{t^{\prime}-t} D^{J}\left(t^{\prime}\right)
$$

and $D^{J}$ as it occurs in Eq. (1.20) is given by Eq. (1.18). This simply states that $f_{ \pm}{ }^{J}(t)$ must have the same phase as $h_{J}(t)$ for $t>4$, but not for $t<t_{0}$.

Before we introduce $f_{ \pm}{ }^{J}$, as given in Eq. (1.19), into Eq. (1.6) and consider the consequences of a modification of the partial-wave sum, it is appropriate to discuss the general properties of the $f_{ \pm}{ }^{J}$ and $h^{J}$. Consider the structure of $h^{J}(t)$. If we could calculate $D^{J}$ in some way, then the $\pi-\pi$ amplitude would be known and any bound states or resonances for a particular value of $J$ in the $\pi-\pi$ amplitude would develop when $\operatorname{Re} D^{J}=0$. Bound states and resonances would appear in the usual way since $\operatorname{Im} D^{J}=0$ for $t<4$. The structure of $f_{ \pm}{ }^{J}$ in Eq. (1.19) shows that such bound states and resonances would also appear in $\pi-e$ scattering, because the same denominator function occurs in both amplitudes. Of course, the situation presented by Eqs. (1.17) to (1.20) is not a realistic one; a proper calculation would involve the coupling between the processes $e+\bar{e} \rightarrow e+\bar{e}, \pi+\pi$ $\rightarrow \pi+\pi$, and $\pi+\pi \rightarrow e+\bar{e}$ and also the $4 \pi$ state. $^{11}$ Such coupled channel problems have been discussed elsewhere, and the crucial point is that the amplitudes have the same general structure as in the simple case.

\footnotetext{
${ }^{11}$ R. Blankenbecler, Phys. Rev. 125, 755 (1962). F. Zachariasen and C. Zemach (unpublished).
} 
Specificially, for an $n$-channel problem, one constructs three $n \times n$ matrices, $\mathbf{M}^{J}, \mathbf{N}^{J}$, and $\mathbf{D}^{J}$ where $\mathbf{M}^{J} \cdot \mathbf{D}^{J}$ $=\mathbf{N}^{J}$. The solutions may be written as ${ }^{8}$

$$
\mathbf{M}^{J}=\mathbf{F}^{J} /\left\|D^{J}\right\|,
$$

where $\mathbf{F}^{J}$ is a function of the $\mathbf{N}^{J}$ and $\mathbf{D}^{J}$ and $\left\|D^{J}\right\|$ $=\operatorname{det} D^{J}$. Thus, all channels will exhibit the same behavior with respect to bound states and resonances, and these are determined by the condition $\operatorname{Re}\left\|D^{J}\right\|=0$. This situation is true for multiple-particle channels as well as two-particle channels. In this connection we remark that the introduction of inelastic channels will always introduce anomalous thresholds, but even in this case $\left\|D^{J}(t)\right\|$ will have only normal cuts ${ }^{12}$ the anomalous-cut behavior appears in $\mathbf{F}^{J}$. Thus, we may consider Eq. (1.19) as a representation of $f_{ \pm}{ }^{J}$ which satisfies the unitarity condition including all possible intermediate states and which has bound states and resonances when $\operatorname{Re}\left\|D^{J}(t)\right\|=0$. The other properties of the process, e.g., size (anomalous thresholds), are given by $r_{ \pm}{ }^{J}$.

Let us return to Eq. (1.6) and introduce Eq. (1.19). We write the partial-wave sum as a contour integral around the positive real axis in the clockwise direction and obtain

$$
\begin{aligned}
& f_{+}{ }^{(o)}\left(t, z_{t}\right)=-\frac{2 \pi i}{p^{2}} \int_{c} d J \frac{(2 J+1)}{\sin \pi J}(p q)^{J^{\prime}} \frac{r_{+}{ }^{J}(t)}{\left\|D^{J}(t)\right\|}\left[P_{J}\left(-z_{t}\right)-P_{J}\left(z_{t}\right)\right] \\
& f_{-}^{(e)}\left(t, z_{t}\right)=-2 \pi i \int_{c} d J \frac{(2 J+1)(p q)^{J-1}}{(\sin \pi J)[J(J+1)]^{1 / 2}} \frac{r_{-}^{J}(t)}{\left\|D^{J}(t)\right\|}\left[P_{J^{\prime}}\left(-z_{t}\right)+P_{J}{ }^{\prime}\left(z_{t}\right)\right]
\end{aligned}
$$

where the definition of $f_{+}{ }^{(o)}\left(t, z_{t}\right)$ and $f_{-}{ }^{(e)}\left(t, z_{t}\right)$ is obvious.

Following the Regge philosophy, we now wish to examine the analytic properties of $r_{ \pm}{ }^{J}(t) /\left\|D^{J}(t)\right\|$ as a function of complex $J$. Specificially, we assume that $f_{ \pm}{ }^{J}(t)$, as a function of complex $J$, has poles, whose positions depend on $t$. If, for a particular $t, \operatorname{Re} J=$ integer and $\operatorname{Im} J=0(\neq 0)$, then $f_{ \pm}{ }^{(0, \theta)}\left(t, z_{t}\right)$ has a bound state (resonance) at that value of $J$ and $t$. Since bound states and resonances are given by zeros in $\left\|D^{J}(t)\right\|$ in the usual analysis, it is very natural to assume that $\left\|D^{J}(t)\right\|$ may be continued into the complex $J$ plane, and has zeros as a function of $J$. In particular, let us assume that $\left\|D^{J}(t)\right\|$ may be factored as

$$
\left\|D^{J}(t)\right\|=d^{J}(t) \prod_{i}\left[J-\alpha_{i}(t)\right]
$$

Supposedly, there are a finite number of $\alpha_{i}$, i.e., $i=1$, $\cdots, N$, compatible with the restriction that they have $I=1$ and baryon number zero which contribute significantly to the scattering amplitude. However, it should be reemphasized that $\left\|D^{J}\right\|$ is, in principle, a denominator function which includes all possible intermediate states. Since we have restricted ourselves to the $I=1$ channel, let us assume that only two trajectories are of interest to us, viz., the photon and $\rho$-meson trajectories. Thus, we approximate Eq. (1.23) as

$$
\left\|D^{J}(t)\right\|=d^{J}(t)\left[J-\alpha_{1}(t)\right]\left[J-\alpha_{2}(t)\right]
$$

where $\alpha_{1}(t)$ and $\alpha_{2}(t)$ are the photon and $\rho$-meson trajectories, respectively, and $d^{J}(t)$ does not have any zeros as a function of $J$ for $\operatorname{Re} J>0$. We return to Eq. (1.22) and use Eq. (1.24) to open the $J$ contours and obtain

$$
\begin{aligned}
& f_{+}{ }^{(o)}\left(t, z_{t}\right)=\frac{4 \pi^{2}}{p^{2}} \frac{2 \alpha_{1}(t)+1}{\sin \pi \alpha_{1}(t)} \frac{(p q)^{\alpha_{1}(t)} \beta_{+}{ }^{(1)}(t)}{\left[\alpha_{1}(t)-\alpha_{2}(t)\right]}\left[P_{\alpha_{1}(t)}\left(-z_{t}\right)-P_{\alpha_{1}(t)}\left(z_{t}\right)\right] \\
& +\frac{4 \pi^{2}}{p^{2}} \frac{2 \alpha_{2}(t)+1}{\sin \pi \alpha_{2}(t)} \frac{(p q)^{\alpha_{2}(t)} \beta_{+}{ }^{(2)}(t)}{\left[\alpha_{2}(t)-\alpha_{1}(t)\right]}\left[P_{\alpha_{2}(t)}\left(-z_{t}\right)-P_{\alpha_{2}(t)}\left(z_{t}\right)\right]+\text { line integral. } \\
& f_{-}{ }^{(e)}\left(t, z_{t}\right)=4 \pi^{2} \frac{2 \alpha_{1}(t)+1}{\sin \pi \alpha_{1}(t)} \frac{(p q)^{\alpha_{1}(t)-1} \beta_{-}{ }^{(1)}(t)}{\left[\alpha_{1}(t)-\alpha_{2}(t)\right]} \frac{\left[P_{\alpha_{1}(t)}{ }^{\prime}\left(-z_{t}\right)+P_{\alpha_{1}(t)}{ }^{\prime}\left(z_{t}\right)\right]}{\left\{\alpha_{1}(t)\left[\alpha_{1}(t)+1\right]\right\}^{1 / 2}} \\
& +4 \pi^{2} \frac{2 \alpha_{2}(t)+1}{\sin \pi \alpha_{2}(t)} \frac{(p q)^{\alpha_{2}(t)-1} \beta_{-}{ }^{(2)}(t)}{\left[\alpha_{2}(t)-\alpha_{1}(t)\right]} \frac{\left[P_{\alpha_{2}(t)}{ }^{\prime}\left(-z_{t}\right)+P_{\alpha_{2}(t)}{ }^{\prime}\left(z_{t}\right)\right]}{\left\{\alpha_{2}(t)\left[\alpha_{2}(t)+1\right]\right\}^{1 / 2}}+\text { line integral, }
\end{aligned}
$$

where

$$
\beta_{ \pm}{ }^{(i)}(t)=r_{ \pm}{ }^{\alpha_{i}(t)}(t) / d^{\alpha_{i}(t)}(t),
$$

and the line integral runs up the imaginary axis to the left of $J=0$. It should be noted that the left-hand cut of $r_{ \pm}{ }^{\alpha_{i}}(t)$ is actually not present because the imaginary part of $r_{ \pm}^{J}$ contains a factor of $D^{J}$ which, of course, vanishes at the Regge pole.

In order to examine the possible effect of the photon and $\rho$-meson trajectories, as opposed to an "elementary"

${ }^{12}$ L. F. Cook and B. W. Lee, Phys. Rev. 127, 283 (1962). 
particle description, we must choose an experimental situation, if possible, where we can neglect the effect of the integral on the amplitude in Eq. (1.25). We follow the customary procedure by considering high-energy scattering in the $e-\pi$ channel of Fig. 1. This will allow an asymptotic expansion of the Legendre functions. For $e-\pi$ scattering we have

$$
s=m^{2}+1-\frac{1}{2} t-2 p q z_{t},
$$

where $p$ and $q$ are still determined as functions of $t$ in the annihilation-channel barycentric system. We may, of course, evaluate $s$ in any Lorentz frame and let us choose the laboratory system of $\pi_{1}$. Then

$$
-z_{t}=\left(E_{L}+\frac{1}{4} t\right) / p q
$$

where $E_{L}$ is the total laboratory energy of the electron. Clearly, if $t$ is to be a physical momentum transfer, it must be negative and if $E_{L}$ is large an asymptotic expansion of the Legendre functions in Eq. (1.25) is allowed. For large $z$, we may write

$$
P_{\alpha}(z)=\frac{2^{\alpha} \Gamma\left(\alpha+\frac{1}{2}\right)}{\pi^{1 / 2} \Gamma(\alpha+1)} z^{\alpha}+\cdots
$$

and

$$
P_{\alpha}(z)=e^{-i \pi \alpha} P_{\alpha}(-z)+O\left(1 / z^{\alpha+1}\right) .
$$

Neglecting the line integral term and writing only the photon trajectory pole for the moment, we obtain

$$
\begin{aligned}
& f_{+}{ }^{(o)}\left(t, E_{L}\right)=\frac{4 \pi^{2}}{p^{2}} \frac{2 \alpha_{1}+1}{\sin \pi \alpha_{1}} \frac{\beta_{+}{ }^{(1)}(t)(2 m)^{\alpha_{1}} \Gamma\left(\alpha_{1}+\frac{1}{2}\right)}{\left[\alpha_{1}-\alpha_{2}\right]}\left(1-e^{-i \pi \alpha_{1}}\right)\left(\frac{E_{L}}{m}+\frac{t}{4 m}\right)^{\alpha_{1} / 2} \Gamma\left(\alpha_{1}+1\right) \\
& f_{-}{ }^{(e)}\left(t, E_{L}\right)=4 \pi^{2} \frac{2 \alpha_{2}+1}{\sin \pi \alpha_{2}} \frac{\beta_{-}{ }^{(1)}(t)}{\left[\alpha_{1}-\alpha_{2}\right]} \frac{(2 m)^{\alpha_{1}-1} \Gamma\left(\alpha_{1}+\frac{1}{2}\right)}{\pi^{1 / 2} \Gamma\left(\alpha_{1}+1\right)} \frac{2 \alpha_{1}}{\left[\alpha_{1}\left(\alpha_{1}+1\right)\right]^{1 / 2}}\left(1-e^{-i \pi \alpha_{1}}\right)\left(\frac{E_{L}}{m}+\frac{t}{4 m}\right)^{\alpha_{1}-1} .
\end{aligned}
$$

It is of interest to compare these expressions with those obtained in first-order perturbation theory in quantum electrodynamics. From Eq. (1.22) we see that

$$
\begin{aligned}
& a^{(o)}\left(t, E_{L}\right)=f_{+}{ }^{(o)}\left(t, E_{L}\right)-m-q q z_{t} f_{-}(e)\left(t, E_{L}\right), \\
& b^{(e)}\left(t, E_{L}\right)=f_{-}{ }^{(e)}\left(t, E_{L}\right) .
\end{aligned}
$$

Using Eqs. (1.30), (1.26), and (1.29), we find

$$
\begin{aligned}
a^{(o)}\left(t, E_{L}\right)= & \frac{4 \pi^{2}}{p^{2}}\left(2 \alpha_{1}+1\right) \frac{(2 m)^{\alpha_{1}}}{\left(\alpha_{1}-\alpha_{2}\right)} \frac{\Gamma\left(\alpha_{1}+\frac{1}{2}\right)}{\pi^{1 / 2} \Gamma\left(\alpha_{1}+1\right)} \\
& \times\left(1-e^{\left.-i \pi \alpha_{1}\right)}\left(\frac{E_{L}}{m}\right)^{\alpha_{1}} \frac{1}{d^{\alpha_{1}(t)}} \frac{\left\|D^{\alpha_{1}}(t)\right\|}{\sin \pi \alpha_{1}}\right. \\
& \times\left[f_{+}{ }^{\alpha_{1}}(t)+p q \frac{\alpha_{1}}{\left[\alpha_{1}\left(\alpha_{1}+1\right)\right]^{1 / 2}} f_{-}^{\alpha_{1}}(t)\right] .
\end{aligned}
$$

Until now we have not had to commit ourselves with regard to the actual behavior of $\alpha_{1}(t)$, but if it is to represent a photon then $\alpha_{1}(0)=1$. Let us evaluate the square bracket in Eq. (1.32) when $t \sim 0$. We need only remark that

$$
\begin{aligned}
& p=\frac{1}{2}\left(t-4 m^{2}\right)^{1 / 2}, \\
& q=\frac{1}{2}(t-4)^{1 / 2},
\end{aligned}
$$

and thus $p q \simeq-m$ when $t \simeq 0$. Therefore

$$
[] \sim f_{+}{ }^{1}(0)-\frac{m}{2^{1 / 2}} f_{-}{ }^{1}(0) \cong 0
$$

in first-order perturbation theory, and $a^{(o)}\left(0, E_{L}\right)=0$ as it should.
In a similar fashion we may examine the structure of $b^{(e)}\left(t, E_{L}\right)$. Let us write $b^{(e)}$ as

$$
\begin{aligned}
b^{(e)}\left(t, E_{L}\right)=\mathcal{P}(t)\left(\frac{E_{L}}{m}+\frac{t}{4 m}\right)^{\alpha_{1}-1}\left(\frac{1-e^{-i \pi \alpha_{1}}}{2}\right) \\
\times \frac{{\frac{2}{1 / 2} 12 \pi^{2}}_{1-\alpha_{2}(0)}^{\beta_{-}^{(1)}(t)}}{\sin \alpha_{1}(t)},
\end{aligned}
$$

where

$$
\rho(t)=\frac{2^{1 / 2}}{3} \frac{\Gamma\left(\alpha_{1}+\frac{1}{2}\right) 2 \alpha_{1}\left(2 \alpha_{1}+1\right)(2 m)^{\alpha_{1}-1}}{\pi^{1 / 2} \Gamma\left(\alpha_{1}+1\right)\left[\alpha_{1}\left(\alpha_{1}+1\right)\right]^{1 / 2}} \frac{1-\alpha_{2}(0)}{\alpha_{1}(t)-\alpha_{2}(t)} .
$$

$P(t)$ may be thought of as the pion form factor and is constructed so that $\rho(0)=1$. Evaluating Eq. (1.34) near $t=0$, we find

$$
b^{(e)}\left(0, E_{L}\right)=\odot(0) \frac{2^{1 / 2} 12 \pi}{1-\alpha_{2}(0)} \frac{\beta_{-}{ }^{(1)}(0)}{\alpha_{1}^{\prime} t},
$$

where $\alpha_{1}(t) \sim 1+\alpha_{1}^{\prime} t$ for $t \sim 0$. Now from Eq. (1.26),

$$
\beta_{-}(1)=\frac{r_{-}^{\alpha_{1}}}{d^{\alpha_{1}}}=f_{-}{ }^{1}(0)\left[1-\alpha_{2}(0)\right] \alpha^{\prime} t,
$$

so that Eq. (1.35) becomes

$$
b^{(e)}\left(0, E_{L}\right)=\odot(0) 2^{1 / 2} 12 \pi f_{-}{ }^{1}(0) .
$$

This should be compared with Eq. (1.6) when only $J=1$ is allowed. Thus, the amplitude, which has been taken to be dominated by a Regge trajectory for the photon, reduces to the conventional form when the quantities are near the position of the field-theoretic pole at $t=0$.

Let us return to the physical interpretation of our 
solutions in Eqs. (1.25) and (1.26). These results are clarified by making a pole approximation for the resonances present in the amplitudes and an "effective range" expansion of the slowly varying numerators. This will yield a modified Clementel-Villi formula ${ }^{13}$ for the form factors. If, in addition, $z_{t}$ is assumed large, then we find

$$
f_{\{ \pm\}}=\frac{e^{2}}{t}\left[a_{ \pm}+\frac{b_{ \pm}+\left(t / t_{2}\right) c_{ \pm} z_{t}^{\alpha_{2}-\alpha_{1}}}{t_{2}-t}\right]\left\{\begin{array}{c}
z_{t}^{\alpha_{1}} \\
z_{t}^{\alpha_{1}-1}
\end{array}\right\}
$$

where $t_{2}$ is the effective $\pi-\pi$ resonance position and $a_{ \pm}, b_{ \pm}$, and $c_{ \pm}$are constants. The interpretation of $a_{ \pm}$, and $b_{ \pm}$is straightforward. The constant $c_{ \pm}$measures the direct coupling of the electron to the pion resonance.

\section{Isovector Electron-Nucleon Scattering}

Our treatment of isovector electron-nucleon scattering will be modeled after the electron-pion case. The general structure of the electron-nucleon matrix element is quite complicated; there are six invariant functions. Our procedure will be to calculate explicitly the absorptive part of the amplitude due to the two-pion intermediate state. This result will serve two purposes. It will provide us with the partial-wave expansion of the electron-nucleon matrix element and will allow an explicit determination of the partial-wave amplitude in the two pion approximation. This latter property will not be directly used since we feel it is essential to break away from the two-particle approximation in any quantitative discussion of the form factors. This is expecially true if the four-pion state has a large effect on the structure of the $T=J=1$ resonance. ${ }^{11}$

The matrix element for the process $N+\bar{N} \rightarrow e+\bar{e}$ can be written as

$$
G\left(t, z_{t}\right)=\bar{u}(p) \bar{V}(\bar{P})\left[\sum_{i} \Theta_{i} G_{i}\left(t, z_{t}\right)\right] U(P) v(\bar{p}),
$$

where $t$ is the square of the center-of-mass energy, $z_{t}$ is the scattering angle, and $u(p)[U(P)]$ labels the spinor and momentum of the electron (nucleon). The matrix invariants have been chosen to be

$$
\begin{aligned}
& \mathcal{\Theta}_{1}=i \gamma^{N} \cdot i \gamma^{e}, \\
& \mathcal{\Theta}_{2}=1^{N} \frac{1}{2}(\bar{P}-P) \cdot i \gamma^{e}, \\
& \mathcal{\Theta}_{3}=i \gamma^{N} \cdot \frac{1}{2}(\bar{p}-p) 1^{e}, \\
& \mathcal{\Theta}_{4}=\frac{1}{2}(\bar{p}-p) \cdot i \gamma^{N} \frac{1}{2}(\bar{P}-P) i \gamma^{e}, \\
& \mathcal{\Theta}_{5}=1^{N} 1^{e}, \\
& \mathcal{\Theta}_{6}=\gamma_{5}{ }^{N} \gamma_{5}{ }^{e} .
\end{aligned}
$$

For fixed $t$ and asymptotically large $z_{t}$ we observe that the expectation values of these matrix invariants

\footnotetext{
${ }^{13}$ See, for example, C. deVries, R. Hofstadter, and R. Herman, Phys. Rev. Letters 8, 381 (1962).
}

between spinors behave like

$$
\begin{aligned}
& \mathcal{O}_{1}, \Theta_{2}, \Theta_{3} \sim z_{t}, \\
& \mathcal{O}_{4} \sim z_{t}{ }^{2}, \\
& \mathcal{O}_{5}, \Theta_{6} \sim 1 .
\end{aligned}
$$

These relations will be of use in discussing the large- $z_{t}$ behavior of the amplitude.

For later comparison purposes, we note that the lowest-order matrix element in quantum electrodynamics is given by

$$
\begin{aligned}
& G_{1}=\left(e^{2} / t\right) g_{1}(t), \\
& G_{2}=\left(e^{2} / t\right) g_{2}(t), \\
& G_{3}=G_{4}=G_{5}=G_{6}=0,
\end{aligned}
$$

where $g_{1}$ and $g_{2}$ are the isovector form factors of the nucleon. The $g$ 's are related to the customary $F$ 's by

$$
\begin{aligned}
& g_{1}=F_{1}+2 M F_{2}, \\
& g_{2}=2 F_{2} .
\end{aligned}
$$

In the two-meson approximation the unitarity condition for $G$ is

$$
\begin{aligned}
\operatorname{Im} G\left(t, z_{t}\right)=\frac{q}{32 \pi^{2} t^{1 / 2}} \int & d \Omega^{\prime} \bar{u}(p)\left(a-i \gamma^{e} \cdot q^{\prime} b\right) v(\bar{p}) \cdot \bar{V}(p) \\
& \times\left(A^{*}-i \gamma^{N} \cdot q^{\prime} B^{*}\right) U(P),
\end{aligned}
$$

where the notation is the same as Eq. (1.7); $a$ and $b$ are defined in Eq. (1.3), and the matrix element for the annihilation of two pions into a nucleon-antinucleon pair is

$$
F=\bar{U}(P)\left[A-i \frac{1}{2}\left(q_{1}-q_{2}\right) \cdot \gamma^{N} B\right] V(\bar{P}) .
$$

The partial-wave expansions of $A$ and $B$ in terms of the angle between $P$ and $q, z^{\prime \prime}$, have the same structure as the electron-pion case; in particular,

$$
\begin{gathered}
F_{+}=A+\left(M q z^{\prime \prime} / P\right) B=-\frac{8 \pi}{P^{2}} \sum_{J}(2 J+1)(P q)^{J} \\
\times F_{+}{ }^{J}(t) P_{J}\left(z^{\prime \prime}\right), \\
\begin{aligned}
F_{-}=B=-8 \pi \sum_{J}(2 J+1)(P q)^{J-1} \\
\quad \times F_{-}{ }^{J}(t) P_{J}{ }^{\prime}\left(z^{\prime \prime}\right) /[J(J+1)]^{1 / 2},
\end{aligned}
\end{gathered}
$$

where

$$
\begin{aligned}
q^{2} & =|\mathbf{q}|^{2}=(t-4) / 4, \\
P^{2} & =|\mathbf{P}|^{2}=\left(t-4 M^{2}\right) / 4 .
\end{aligned}
$$

Since we are only concerned with $I=1$, only $A^{(o)}$ and $B^{(e)}$ will contribute and we could write Eq. (2.7) as Eq. (1.6), but because of the notational complications we will explicitly include the signature later. The unitarity condition for the $F_{ \pm}{ }^{J}$, again in the two-pion approximation, is the same as that found earlier for the $f_{ \pm}{ }^{J}$, specifically,

$$
\operatorname{Im} F_{ \pm}^{J}=\left(q^{2 J+1} / t^{1 / 2}\right) h_{J}^{*} F_{ \pm}^{J} \theta(t-4) .
$$


In order to carry out the angular integration involved in the unitarity relation, Eq. (2.5), it is convenient to expand the gamma matrices occurring in $F$ and $f$ in a set of vectors defined by

$$
\begin{aligned}
\boldsymbol{\gamma}^{N}= & \mathbf{P}\left[\boldsymbol{\gamma}^{N} \cdot \mathbf{P} / P^{2}\right]+\left(\mathbf{p}-\mathbf{P} \frac{\mathbf{P} \cdot \mathbf{p}}{P^{2}}\right) \\
& \times\left\{\boldsymbol{\gamma}^{N} \cdot\left(\mathbf{p}-\mathbf{P} \frac{\mathbf{P} \cdot \mathbf{p}}{P^{2}}\right) /\left[p^{2}-(\mathbf{P} \cdot \mathbf{p})^{2} / P^{2}\right]\right\} \\
& +(\mathbf{p} \times \mathbf{P})\left\{\boldsymbol{\gamma}^{N} \cdot(\mathbf{p} \times \mathbf{P}) /\left[p^{2} P^{2}-(\mathbf{P} \cdot \mathbf{p})^{2}\right]\right\} \\
\boldsymbol{\gamma}^{e}=\boldsymbol{\gamma}^{N} & \left(\boldsymbol{\gamma}^{N} \rightarrow \boldsymbol{\gamma}^{e}, \mathbf{P} \leftrightarrow \mathbf{p}\right) .
\end{aligned}
$$

Now using the fact that between the spinors involved in our problem, one may make the replacement $\boldsymbol{\gamma}^{N} \cdot \mathbf{P}$ $=i M$ and $\boldsymbol{\gamma}^{e} \cdot \mathbf{p}=i m$, the unitarity integral can be written in a considerably more tractable form. These latter integrals can be carried out by elementary means. If the term involving $\boldsymbol{\gamma}^{N} \cdot(\mathbf{p} \times \mathbf{P}) \boldsymbol{\gamma}^{e} \cdot(\mathbf{p} \times \mathbf{P})$ is eliminated in favor of $\boldsymbol{\gamma}^{N} \cdot \boldsymbol{\gamma}^{e}$ by use of Eqs. (2.10) and (2.11), one may obtain directly the partial-wave expansion of the invariant functions introduced in Eqs. (2.1) and (2.2). The result is

$$
\begin{gathered}
G_{1}\left(t, z_{t}\right)=\sum_{J}(2 J+1)(P p)^{J-1} G_{1}{ }^{J}(t) P_{J}{ }^{\prime}\left(z_{t}\right)[J(J+1)]^{-1 / 2}, \\
G_{2}\left(t, z_{t}\right)=\sum_{J}(2 J+1) \frac{(P p)^{J-1}}{P^{2}}\left[-\frac{M G_{1}{ }^{J}}{J(J+1)}\left(P_{J^{\prime}}+z_{t} P_{J^{\prime}}{ }^{\prime \prime}\right)\right. \\
\left.+\frac{G_{2}{ }^{J}}{[J(J+1)]^{1 / 2}} P_{J^{\prime}}\left(z_{t}\right)\right], \\
G_{3}\left(t, z_{t}\right)=\sum_{J}(2 J+1) \frac{(P p)^{J-1}}{p^{2}}\left[-\frac{m G_{1}{ }^{J}}{J(J+1)}\left(P_{J^{\prime}}+z_{t} P_{J^{\prime \prime}}\right)\right. \\
\left.\quad+\frac{G_{3}{ }^{J}}{[J(J+1)]^{1 / 2}} P_{J^{\prime}}\left(z_{t}\right)\right], \quad(2.12) \\
G_{4}\left(t, z_{t}\right)=\sum_{J}(2 J+1)(P p)^{J-2} G_{1}{ }^{J} P J^{\prime \prime}\left(z_{t}\right) / J(J+1), \\
G_{5}\left(t, z_{t}\right)=\sum_{J}(2 J+1)(P p)^{J-2}\left[\frac{M m G_{1}{ }^{J}}{J_{(J+1)}} z_{t}\left(P_{J^{\prime}}+z_{t} P_{J^{\prime}}{ }^{\prime \prime}\right)\right. \\
\left.G_{6}\left(t, z_{t}\right)=0 . \quad-\frac{m G_{2}{ }^{J}+M G_{3}{ }^{J}}{[J(J+1)]^{1 / 2}} z_{t} P_{J}{ }^{\prime}+G_{4}{ }^{J} P_{J}\right],
\end{gathered}
$$

In addition, one finds for the unitarity conditions on the $G$ 's :

$$
\begin{aligned}
& \operatorname{Im} G_{1}{ }^{J}=\left(8 \pi q^{2 J+1} / t^{1 / 2}\right) F_{-}{ }^{J *} f_{-}{ }^{J}, \\
& \operatorname{Im} G_{2}{ }^{J}=\left(8 \pi q^{2 J+1} / t^{1 / 2}\right) F_{+}{ }^{J *} f_{-}{ }^{J}, \\
& \operatorname{Im} G_{3}{ }^{J}=\left(8 \pi q^{2 J+1} / t^{1 / 2}\right) F_{-}{ }^{*} f_{+}{ }^{J}, \\
& \operatorname{Im} G_{4}{ }^{J}=\left(8 \pi q^{2 J+1} / t^{1 / 2}\right) F_{+}{ }^{J *} f_{+}{ }^{J} .
\end{aligned}
$$

If unsubtracted dispersion relations are assumed for $G_{3}, G_{4}$, and $G_{5}$, then in the limit of first-order perturbation theory where the photon is taken as elementary (all $f_{-} J=0$ if $J \neq 1$ ), $a$ vanishes because

and one finds

$$
f_{+}{ }^{1}-(m / \sqrt{2}) f_{-}{ }^{1}=0,
$$

and also

$$
G_{3}=G_{4}=G_{5}=0,
$$

$$
\begin{aligned}
& \operatorname{Im} G_{1}=\frac{3}{2}\left(8 \pi q^{2 J+1} / t^{1 / 2}\right) F_{-}{ }^{*} f_{-}{ }^{1} \\
& \operatorname{Im} G_{2}=\frac{3}{2}\left(8 \pi q^{2 J+1} / t^{1 / 2}\right)\left(1 / P^{2}\right)\left(\sqrt{2} F_{+}{ }^{1}-M F_{-}\right)^{*} f_{-}{ }^{1},
\end{aligned}
$$

which agrees with the decomposition of Frazer and Fulco.

The partial-wave decomposition of the $G$ 's given by Eq. (2.12) is, of course, not a general one, since for each value of $J$ there must be six $G^{J}$ 's. The remaining two $G^{J}$ 's correspond to the exchange of states with negative " $G$ parity." The additional contributions to the invariants coming from such states are:

$$
\begin{aligned}
G_{1}\left(t, z_{t}\right) & =\sum(2 J+1)(P p)^{J} \frac{G_{5}{ }^{J}(t)}{J(J+1)} \\
\times\left[-z_{t} P P^{\prime}+J(J+1) P_{J}\right] & \\
G_{2}\left(t, z_{t}\right)= & \sum(2 J+1)(P p)^{J} \frac{M}{P^{2}} \frac{G_{5}{ }^{J}}{J(J+1)} P_{J^{\prime \prime}}, \\
G_{3}\left(t, z_{t}\right)= & \sum(2 J+1)(P p)^{J} \frac{m}{p^{2}} \frac{G_{5}{ }^{J}}{J(J+1)} P_{J^{\prime \prime}}, \\
G_{4}\left(t, z_{t}\right)= & -\sum(2 J+1)(P p)^{J-1} \frac{G_{5}{ }^{J}}{J(J+1)}\left[P_{\left.J^{\prime}+z_{t} P_{J^{\prime}}\right]},\right. \\
G_{5}\left(t, z_{t}\right)= & -\sum(2 J+1)(P p)^{J-1} \frac{G_{5}{ }^{J}}{J(J+1)} m M_{z_{t}} P_{J^{\prime}}{ }^{\prime \prime}, \\
G_{6}\left(t, z_{t}\right)= & \sum(2 J+1)(P p)^{J} G_{6}{ }^{J} P_{J} .
\end{aligned}
$$

For example, if we treat the $\pi^{0}$ meson as an elementary particle (coupled to the electron via two photons, say), its exchange would lead to an amplitude of the form $\Theta_{6}\left(m_{\pi}^{2}-t\right)^{-1}$ and hence

$$
G_{1}{ }^{J}, \cdots, G_{5}{ }^{J}=0 ; \quad G_{6}{ }^{J} \sim \delta_{J_{0}} /\left(m_{\pi}^{2}-t\right) .
$$

Similarly, the exchange of the pseudovector particle suggested by Dennery and Primakoff ${ }^{14}$ gives rise to an amplitude

$\left[i \gamma_{\mu}{ }^{e} \gamma_{5}{ }^{e} i \gamma_{\mu}{ }^{N} \gamma_{5}{ }^{N}+\frac{i \gamma^{e} \cdot(p+\bar{p}) \gamma_{5}{ }^{e} i \gamma^{N} \cdot(P+\bar{P}) \gamma_{5}^{N}}{m_{D}{ }^{2}}\right] \frac{1}{m_{D}{ }^{2}-t}$, where $m_{D}$ is the mass. Expressing this in terms of our

${ }^{14} \mathrm{P}$. Dennery and H. Primakoff, Phys. Rev. Letters 8, 350 (1962). 
standard invariants, we find

$G_{1}{ }^{J}, \cdots G_{4}{ }^{J}=0 ; \quad G_{5}^{J} \sim \frac{8}{3} \frac{\delta_{J 1}}{m_{D}{ }^{2}-t} \frac{1}{t}, \quad G_{6}{ }^{J} \sim-4 \delta_{J 0} \frac{m M}{m_{D}{ }^{2} t}$.

There are two remarks that should be made about the foregoing results. First, the apparent singularity at $t=0$ is of purely kinematic origin and, of course, does not lead to an infinite amplitude in the forward direction. Second, it would appear that the partial-wave amplitudes for both $G_{1}$ and $G_{4}$ behave in an anomalous manner for large $z_{t}$, i.e., $z_{t}^{J+1}$. There is a cancellation between these two contributions to the total amplitude which ultimately goes as $z_{t}^{J}$. This may be made manifest by choosing as a basic invariant $i \gamma^{e} \cdot(\bar{P}-P) \gamma_{5}{ }^{e} i \gamma^{N}$. $(\bar{p}-p) \gamma_{5}{ }^{N}$ instead of $\mathcal{\Theta}_{1}$. (The normal one-photon exchange then becomes rather involved.)

These states with negative $G$ parity are quite different from the ones usually considered in discussing the isovector nucleon structure. We have no assurance that their effects are negligible but one feels intuitively that their contributions are less important than those arising from the states with the quantum numbers of two pions (which after all contain the photon pole). In order to simplify the formulas we shall set $G_{5}{ }^{J}=G_{6}{ }^{J}$ $=0$ and later indicate how the results are modified by including them.

We now face the problem of solving the coupled nucleon-pion, pion-pion, and pion-electron problem. Our task is to solve these coupled systems for the electron-nucleon scattering amplitudes $G$. To this end, the matrix $N / D$ formulation ${ }^{8}$ is introduced, where the channels are labeled by

$$
\begin{aligned}
& 1=e+\overline{\mathrm{e}}, \\
& 2=\pi+\pi, \\
& 3=N+\bar{N} .
\end{aligned}
$$

As an example of this technique, the function $G_{1}{ }^{J}$ will be considered first. The rest of the $G^{J}$ 's will follow in a similar manner. The unitarity relations given by (1.16), (2.9), and (2.18) suggest that we consider a scattering matrix $\mathbf{M}^{J}$ whose elements of interest are given by

$$
\begin{aligned}
& M_{12}{ }^{J}=M_{21}{ }^{J}=f_{-}{ }^{J}, \\
& M_{13}{ }^{J}=M_{31}{ }^{J}=G_{1}{ }^{J}, \\
& M_{22}{ }^{J}=h^{J} / 8 \pi .
\end{aligned}
$$

The unitarity condition satisfied by $\mathbf{M}$ is

$$
\operatorname{Im} \mathbf{M}^{J}=\mathbf{M}^{J} \mathbf{0}^{J} \mathbf{M}^{J *}
$$

where, in the two-pion approximation,

$$
\mathbf{e}^{J}=\left(\begin{array}{ccc}
0 & 0 & 0 \\
0 & \rho_{2}{ }^{J} & 0 \\
0 & 0 & 0
\end{array}\right)
$$

and

$$
\rho_{2}{ }^{J}=8 \pi q^{2 J+1} / t^{1 / 2} .
$$

One can, of course, do much better than this simple approximation by retaining all the diagonal elements of $\mathbf{0}^{J}$. However, this simplified model will give the structure of the matrix $M$ which is all we are interested in for our present purposes. In order to solve the unitarity equation (2.23), one introduces a matrix $\mathbf{N}^{J}$ which has only left-hand singularities and a matrix $\mathrm{D}^{J}$ defined by

$$
\mathbf{D}^{J}=1-\int_{4}^{\infty} \frac{d t^{\prime}}{\pi} \frac{1}{t^{\prime}-t} \mathbf{Q}^{J}\left(t^{\prime}\right) \mathbf{N}^{J}\left(t^{\prime}\right)
$$

The question of subtractions will be taken up later. One finds that the matrix

$$
\mathbf{M}^{J}=\mathbf{N}^{J}\left(\mathbf{D}^{J}\right)^{-1}
$$

satisfies (2.15), the unitarity condition.

In order to be consistent with our previous results for the pion-electron, pion-pion, and nucleon-pion amplitudes, we define

and

$$
\begin{gathered}
N_{12}{ }^{J}=r_{-}{ }^{J}, \quad N_{32}{ }^{J}=R_{-}{ }^{J}, \quad N_{22}{ }^{J}=n^{J}, \\
D_{22}{ }^{J} N_{21}{ }^{J}=r_{-}{ }^{J}+n^{J} D_{21}{ }^{J}, \\
D_{22}{ }^{J} N_{23}{ }^{J}=R_{-}{ }^{J}+n^{J} D_{23}{ }^{J} .
\end{gathered}
$$

Further, we make the unessential assumption that the $G$ 's do not require subtractions and choose

$$
N_{13}{ }^{J}=N_{31}{ }^{J}=0 .
$$

These definitions lead to

$$
\begin{gathered}
h^{J}=q^{2 J} n^{J} / D_{22}{ }^{J}, \\
f_{-}{ }^{J}=r_{-}{ }^{J} / D_{22}{ }^{J}, \\
F_{-}{ }^{J}=R_{-}{ }^{J} / D_{22}{ }^{J},
\end{gathered}
$$

and

$$
G_{1}{ }^{J}=-R{ }^{J} D_{21}{ }^{J} / D_{22}{ }^{J}=-r_{-}{ }^{J} D_{23}{ }^{J} / D_{22}{ }^{J} .
$$

The solutions for the other $G$ 's are of the same form but contain the appropriate combinations of $r_{ \pm}{ }^{J}$ and $R_{ \pm}^{J}$ required by (2.19).

Just as in the pion-electron case, this solution for the $G$ 's may be formally extended to more channels, i.e., the four-pion channel, by slightly changing the definition of the numerators and regarding the denominator function $D_{22}{ }^{J}$ as the determinant of the complete $\mathrm{D}^{J}$ matrix. ${ }^{12}$

Our next step is to use these explicit solutions for the $G^{J}$ 's to achieve a Regge representation of the nucleonelectron scattering amplitude. The procedure is the same as in Sec. 1 and the factorized form (1.24) for $D_{22}{ }^{J}$ will be used. The results for $G_{1}$ and $G_{2}$ are

$$
G_{1}\left(t, z_{t}\right)=\left\{\left[\frac{P_{\alpha_{1}}{ }^{\prime}\left(z_{t}\right)+P_{\alpha_{1}}{ }^{\prime}\left(-z_{t}\right)}{2 \sin \pi \alpha_{1}}\right] \frac{\pi L_{-}{ }^{\alpha_{1}}(t)}{\left(\alpha_{1}-\alpha_{2}\right) d^{\alpha_{1}}}\left[\alpha_{1}\left(\alpha_{1}+1\right)\right]^{1 / 2}\right\}+\left\{\alpha_{1} \leftrightarrow \alpha_{2}\right\},
$$




$$
\begin{aligned}
G_{2}\left(t, z_{t}\right)=\left\{\left[\frac{P_{\alpha_{1}}{ }^{\prime}\left(z_{t}\right)+P_{\alpha_{1}}{ }^{\prime}\left(-z_{t}\right)}{2 \sin \pi \alpha_{1}}\right.\right. & ] \frac{\pi L_{+}{ }^{\alpha_{1}}(t)}{\left(\alpha_{1}-\alpha_{2}\right) d^{\alpha_{1}}} \frac{\left[\alpha_{1}\left(\alpha_{1}+1\right)\right]^{1 / 2}}{P^{2}} \\
+ & {\left.\left[\frac{P_{\alpha_{1}}{ }^{\prime}\left(z_{t}\right)+P_{\alpha_{1}}{ }^{\prime}\left(-z_{t}\right)}{2 \sin \pi \alpha_{1}}+z_{t} \frac{P_{\alpha_{1}}{ }^{\prime \prime}\left(z_{t}\right)-P_{\alpha_{1}}{ }^{\prime \prime}\left(-z_{t}\right)}{2 \sin \pi \alpha_{1}}\right] \frac{\pi M L_{-} \alpha_{1}(t)}{\left(\alpha_{1}-\alpha_{2}\right) d^{\alpha_{1}} P^{2}}\right\}+\left\{\alpha_{1} \leftrightarrow \alpha_{2}\right\}, }
\end{aligned}
$$

where

$$
L_{ \pm}^{J}(t)=-\frac{2 J+1}{J(J+1)}(P p)^{J-1} R_{ \pm}{ }^{J}(t) D_{21}{ }^{J}(t) .
$$

It is to be noted that the left-hand singularities of $R_{ \pm}{ }^{\alpha_{1}}, R_{ \pm}{ }^{\alpha_{2}}$ vanish, since they are proportional to $D_{22^{\alpha}}, D_{22}{ }^{\alpha_{2}}$.

We have not yet committed ourselves to any definite subtraction philosophy. It is easily seen that the quantities in $G_{1}$ do not require any subtractions whereas in $G_{2}$, the kinematical singularity due to the factor of $\left(P^{-2}\right)$ is most easily taken care of by subtracting $D_{21}{ }^{J}$ so that it vanishes at $t=4 M^{2}$. Similar subtractions will take case of the kinematical singularities in the other $G$ 's.

The physical interpretation of these results is best illustrated by taking the large $z_{t}$ limit, replacing the resonant denominators by simple poles and approximating the slowly varying numerators by linear functions of $t$ just as before. The results for $G_{1}$ and $G_{2}$ can be written in the form

$$
G_{i}=\frac{1}{t}\left[A_{i}+\frac{B_{i}+C_{i} z^{\alpha_{2}-\alpha_{1}}\left(t / t_{2}\right)}{\left(t_{2}-t\right)}\right] z_{t}{ }^{\alpha_{1}-1},
$$

where $z_{t} \sim\left[2 E /(-t)^{1 / 2}\right]$ and $t_{2}$ is the position of the two-meson vector resonance, $\operatorname{Re} \alpha_{2}\left(t_{2}\right)=1$. This form is to be contrasted with the conventional ClementelVilli'is formula which has $C_{i}=0$ and $\alpha_{1}=1$. We note again that if the photon is elementary, all $G$ 's other than $G_{1}$ and $G_{2}$ are zero. Finally, it follows from (2.3) and the Regge form of (2.12) that the entire amplitude behaves like

$$
G\left(t, z_{t}\right) \sim_{t^{\alpha}}^{\alpha_{1}} \mathcal{G}(t)
$$

for very large $z_{t}$, since we expect $\alpha_{1}(t)>\alpha_{2}(t)$ for all $t$.

Returning briefly to the negative $G$-parity states, we note that the trajectory containing the $\pi^{0}$ meson would contribute a term to $G_{6}$ which behaves like $z_{t}{ }^{\alpha_{0}(t)}$ where $\alpha_{0}\left(m_{\pi}^{2}\right)=0$, in contrast to $\alpha_{1}(0)=1$; for large $z_{t}$ the effect of the $\pi^{0}$ should be negligible. It is less easy to dispose of the pseudovector meson trajectory since $\alpha\left(m_{D}{ }^{2}\right)=1$. It would give rise to a term in Eq. (2.24) with exactly the same structure as that coming from the $\rho$ trajectory, and could be easily included.

\section{Isoscalar Electron-Nucleon Scattering}

The lowest mass state contributing to the isoscalar amplitude is the three-pion state. Since there does not exist any firm theoretical foundation on which to base a dynamical calculation of this state, we prefer to carry out our discussion by analogy with the isovector case. It is clear that the kinematical discussion given in Sec. B 2 will carry over to the three-pion state. The partial-wave expansions of the $G$ 's is of special interest to us and will still be given by Eq. (2.12). The unitarity conditions Eq. (2.13) will be changed because there will still be integrations over the remaining phase space variables need to specify the three particle state. It follows from the generalized $N / D$ method (and in this connection see reference 12), that the solutions for the $G$ 's will be in the form

$$
G_{i}{ }^{J}(t)=s_{i}{ }^{J}(t) / D^{J},
$$

where $D^{J}$ is the determinant of the $D$ matrix for the isoscalar channel.

In order to achieve a Regge representation of the scattering amplitude, we assume that $D^{J}$ can be written as

$$
D^{J}=\left(J-\alpha_{1}\right)\left(J-\alpha_{3}\right) d^{J}(t),
$$

where $\alpha_{3}$ is the Regge trajectory that includes the isoscalar three-pion resonance, $\operatorname{Re} \alpha_{3}\left(t_{3}\right)=1$. We have assumed in Eq. (3.2) that the isoscalar photon trajectory $\alpha_{1}(t)$ is the same as the isovector one. There is no reason to believe this to be the case except, of course, they both must be unity at $t=0$. In this connection it would be extremely interesting to compare the results of electron-helium (or deuteron) elastic scattering with electron-proton scattering. In the former case only isoscalar photons are involved, whereas in the photon the isovector interaction also appears. Needless to say, electron-pion scattering would be a much cleaner experiment, since as we shall see, the electron proton amplitude is extremely complicated. Since we do not anticipate a dramatic difference between the two photon trajectories, we shall in the subsequent formulas take them to be the same. It would be trivial to take any difference into account.

The scalar form factors in the same limit that led to the Clementel-Villi form (2.24) in the vector case are

$$
G_{i}=\frac{1}{t}\left[a_{i}+\frac{b_{i}+c_{i} z_{t}^{\alpha_{3}-\alpha_{1}}\left(t / t_{3}\right)}{\left(t_{3}-t\right)}\right] z_{t}^{\alpha_{1}-1}
$$

Again all the other $G$ 's are zero if the photon is elementary. If the photon is a Regge pole, then the other $G$ 's are nonzero and $G_{4}$ and $G_{6}$ are not singular at $t=0$. 


\section{Electron-Helium Scattering}

There are two aspects of electron-helium scattering which require some discussion. The first point is that there are anomalous thresholds present in the $t$ variable due to the weak binding of the nucleons. This problem will be handled by an analytic continuation in the helium mass starting with a small value. The second aspect is the fact that only the isoscalar part of the electron interaction contributes. This channel has the three-pion state and the nucleon-pair state as its principal contributions. The three-pion state will be handled as in the previous section. The nucleon-pair state will be used to determine the purely geometrical aspects of the helium form factors. The coupled channel problem between the helium-electron and nucleonelectron amplitudes will be formulated and solved. This solution can then be directly extended to include the three-pion state. The Regge representation will then follow as before.

The amplitude for an alpha-particle pair to produce an electron-positron pair will be written as

$$
M_{14}=\bar{u}(p)\left[H_{1}-i \frac{1}{2} \gamma^{e} \cdot(h-\bar{h}) H_{2}\right] v(\bar{p}),
$$

where $h$ is the four-momentum of the helium. The partial-wave expansions for $H_{1}$ and $H_{2}$ in the center-ofmass system have the same structure as the pionelectron case:

$$
\begin{gathered}
H_{+}=H_{1}+\frac{m h}{p} z_{t} H_{2}=-\frac{8 \pi}{p^{2}} \sum(2 J+1)(h p)^{J} \\
\times H_{+}{ }^{J}(t) P_{J}\left(z_{t}\right), \\
H_{-}=H_{2}=-8 \pi \sum(2 J+1)(h p)^{J-1} \\
\times H_{-}{ }^{J} P_{J}{ }^{\prime}\left(z_{t}\right)[J(J+1)]^{-1 / 2},
\end{gathered}
$$

where $h^{2}=\frac{1}{4} t-M_{4}^{2}, M_{4}$ is the helium mass and $m_{e}$ is the electron mass. Our first task is to determine the functions $H_{ \pm}{ }^{J}$ by the unitarity condition in the twonucleon approximation. This result will be then extended to include all states, for example, the three-pion state.

The amplitude for nucleon-pair production by the annihilation of an alpha-particle pair will be written as

$$
M_{34}=\bar{U}(P)\left[\mathfrak{K}_{1}-i \frac{1}{2} \gamma^{N} \cdot(h-\bar{h}) \mathfrak{H}_{2}\right] V(\bar{P}),
$$

where $\mathfrak{H}_{1}$ and $\mathfrak{H}_{2}$ have the partial-wave expansion

$$
\begin{aligned}
\mathcal{H}_{+}=\mathcal{H C}_{1}+\frac{M h}{P} z_{t} \mathcal{H C}_{2} & =-\frac{8 \pi}{P^{2}} \sum(2 J+1)(h p)^{J} \\
\times \mathcal{H}_{+}{ }^{J}(t) P_{J}\left(z_{t}\right) & \\
\mathcal{H}_{-}=\mathcal{H C}_{2} & =-8 \pi \sum(2 J+1)(h P)^{J-1} \\
& \times \mathcal{H C}_{-} J(t) P_{J}\left(z_{t}\right)[J(J+1)]^{-1 / 2},
\end{aligned}
$$

and $P^{2}=\frac{1}{4} t-M^{2}$.
The unitarity condition for $M_{14}$ is

$$
\operatorname{Im} M_{14}=\frac{P}{32 \pi^{2} t^{1 / 2}} \sum_{\text {spins }} \int d \Omega_{P} M_{13} * M_{34},
$$

where $M_{13}$ is the electron-nucleon amplitude (2.1). The sum over nucleon spins may be turned into a trace which is easily evaluated. The angular integral may be carried out by using the same method as in Sec. B 2 . The results for $H_{ \pm}{ }^{J}$ are

$$
\begin{aligned}
& \operatorname{Im} H_{-}{ }^{J}(t)=\frac{P^{2 J-1}}{4 \pi M^{2} t^{1 / 2}}\left[\mathcal{H}_{+}{ }^{*} G_{2}{ }^{J}+\stackrel{t}{-\mathcal{H}_{-} J * G_{1}}{ }_{4}\right] \\
& \operatorname{Im} H_{+}{ }^{J}(t)=\frac{P^{2 J-1}}{4 \pi M^{2} t^{1 / 2}}\left[\mathcal{H}_{+} J * G_{4}{ }^{J}+\underset{4}{t} \mathcal{H C}_{4} J * G_{3}{ }^{J}\right] \text {. }
\end{aligned}
$$

If the electron has no anomalous moment, specificially $a=0$, and the photon is elementary, then only $J=1$ terms are nonzero, and $G_{4}{ }^{J}=G_{3}{ }^{J}=0$. Hence $H_{+}{ }^{J}=0$, as expected.

The solutions of the unitarity conditions (4.8) and (4.9) together with analogous unitarity expressions for $\mathcal{H}_{ \pm}{ }^{J}$ and $G_{i}{ }^{J}$ are

$$
\begin{aligned}
& H_{-}^{J}(t)=-\left[G_{2}{ }^{J} D_{34}{ }^{J+}+G_{1}{ }^{J} D_{34}{ }^{J-}\right], \\
& H_{+}{ }^{J}(t)=-\left[G_{4}{ }^{J} D_{34}{ }^{J+}+G_{3}{ }^{J} D_{34}{ }^{J-}\right],
\end{aligned}
$$

where

$$
D_{34}{ }^{J \pm}=-\int_{4 M^{2}}^{\infty} \frac{d t^{\prime}}{\pi} \frac{1}{\left(t^{\prime}-t\right)} \frac{\left(P^{\prime}\right)^{2 J-1}}{4 \pi M^{2}\left(t^{\prime}\right)^{1 / 2}} N_{34}{ }^{J \pm}\left(t^{\prime}\right) .
$$

If we were to include other intermediate states, such as the all important three-pion contribution, only the numerator of $H_{ \pm}{ }^{J}$ would be affected. There would still be an over-all factor of $1 / D^{J}$ as defined in Eq. (3.1).

The factor $N_{34} J_{ \pm}$is essentially the coupling between the nucleon pair and the helium pair states. It takes the form of an integral over a left-hand cut:

$$
N_{34}{ }^{J \pm}(t)=\int_{-\infty}^{t_{0}} \frac{d t^{\prime}}{\pi} \frac{L_{ \pm}^{J}\left(t^{\prime}\right)}{\left(t^{\prime}-t\right)} .
$$

For details of this type of result and the following discussion, see reference 15 . The value of $t_{0}$ is less than $4 M^{2}$ if the mass of the helium, $M_{4}$, is small enough, and this is assumed to be the case. The value of $t_{0}$ is found by examining the graph shown in Fig. 3. It turns out to be

$$
t_{0}=\frac{1}{M_{3}^{2}}\left[\left(M_{3}+M\right)^{2}-M_{4}^{2}\right]\left[M_{4}^{2}-\left(M_{3}-M\right)^{2}\right],
$$

where $M_{3} \simeq 3 M$, or more exactly, the triton or helium three mass, and $M_{4}=4 M-\epsilon_{4}$.

${ }^{15}$ R. Blankenbecler, M. Goldberger, S. MacDowell, and S. Treiman, Phys. Rev. 123, 692 (1961). 
TABLE I. Kinematic variables that correspond to $z_{t}=5$ for electron-nucleon scattering.

\begin{tabular}{rcc}
\hline$t\left(\mu^{2}\right)$ & $E_{\text {lab }}(\mathrm{MeV})$ & $\theta_{\text {lab }}$ \\
\hline-200 & 8200 & $16^{\circ}$ \\
-100 & 4860 & $18^{\circ}$ \\
-50 & 3040 & $20^{\circ}$ \\
-10 & 1180 & $22^{\circ}$ \\
-5 & 810 & $23^{\circ}$ \\
\hline
\end{tabular}

The essential point in the continuation to the physical helium mass is that the branch point $t_{0}$ hooks around the point $4 M^{2}$ (when $M_{4}^{2} \sim 10 M^{2}$ ) and thus forces a deformation of the line integral used to define $D_{34}{ }^{J}$. This leads to an anomalous threshold at the point $t_{0} \simeq(32 / 3) M \epsilon_{4}$ in the $H_{ \pm}{ }^{J}(t)$ functions corresponding to the large structure effects due to the weak binding. The crucial point we are stating here is that the anomalous threshold should not affect the Regge procedure. The only change from the normal case is the presence of structure singularities in the residue of the Regge poles. To prove this fact, we must perform a Watson-Sommerfeld transformation on the $H_{1}$ and $H_{2}$ functions. In the pole approximation, the result takes a slightly different form from that discussed elsewhere. This is due to the fact that the anomalous threshold present in $D_{34}$ precludes any polynomial expansion of these rapidly varying factors along the anomalous cut.

The remaining factor in the numerator of $H_{ \pm}$is $s^{J}(t)$ which has a negative cut in $t$, and whose discontinuity is proportional to $\mathscr{D}^{J}$ even in the many-channel case. Therefore, the residue of the Regge pole is analytic below $t_{0}$ since its imaginary part vanishes there.

Our final result takes the form

$$
\begin{aligned}
& H_{-}(t) \\
& \quad=\frac{1}{t}\left[Q_{-}(t)+\frac{\beta_{-}(t)+\mathcal{e}_{-}(t) z_{t}^{\alpha_{3}-\alpha_{1}}\left(t / t_{3}\right)}{\left(t_{3}-t\right)}\right] z_{t}^{\alpha_{1}-1},
\end{aligned}
$$

and a similar result holds for $H_{+}{ }^{J}$ except that the pole at $t=0$ is missing if the electron has no anomalous moment. The functions $\mathscr{Q}, \mathbb{B}$, and $\mathbb{C}$ are essentially the structure factors for helium. They contain the anomalous threshold effects so that they are cut from $t_{0}$ to infinity. We may note at this point that the conventional approximation is to assume that the helium form factor is actually a product of the true helium-structure factor times the scalar nucleon-structure factor. Thus, we see that (4.15) would become

$$
H_{-}(t)=\frac{H(t)}{t}\left[a+\frac{b+c z_{t}^{\alpha_{3}-\alpha_{1}}\left(t / t_{3}\right)}{t_{3}-t}\right] z_{t}^{\alpha_{1-1}}
$$

\section{CONCLUSION}

\section{Results}

In general, the amplitude for electron scattering is a complicated function of both energy and momentum
FIg. 3. Diagram leading to anomalous threshold in $\mathrm{He}^{4}-\left(\right.$ anti- $\left.\mathrm{He}^{4}\right)$ annihilation to $N \bar{N}$.

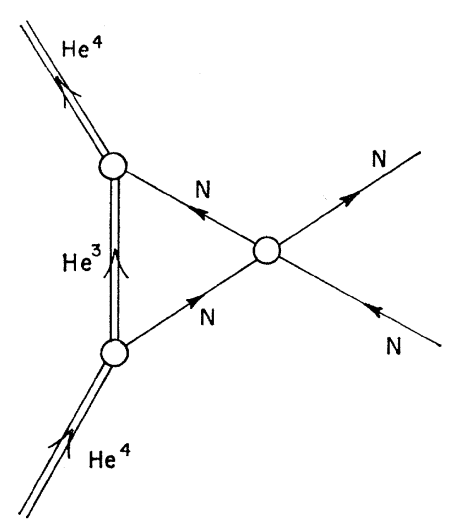

transfer. In the conventional analysis, where one assumes that the photon is elementary, and that only one-photon exchange processes need be considered, the amplitude may be expressed in terms of form factors (functions of momentum transfer only) and trivial kinematic energy factors. At high energies and large momentum transfers, one expects this analysis to break down because of dispersive effects associated with multiple photon exchange. If we make the Regge hypothesis, however, there is a rather natural way to extend the form factor analysis into the dispersive regime; a crucial test of this hypothesis seems experimentally feasible.

We have seen in the previous section that the scattering amplitude will be dominated by the Regge pole contributions provided that the quantity $z_{t}$ is sufficiently large compared to unity. In general, this will be true for high energies and sufficiently small momentum transfers. To illustrate the experimental restrictions we show in Table I the various energies which, for the indicated momentum transfers, lead to the value $z_{t}=5$. (The table was computed for electronnucleon scattering.) The angle $\theta$ is the corresponding lab angle. At a fixed momentum transfer, as the energy is increased, our approximations get better, since $z_{t}$ also increases.

Let us consider the case of electron-helium scattering. In the laboratory system, the cross section is

$$
\begin{aligned}
\frac{d \sigma}{d \Omega}=\left(\frac{E^{\prime}}{8 \pi M_{4} E}\right)^{2} & \\
& \times\left[-t\left|H_{+}\right|^{2}+\left|H_{-}\right|^{2} \frac{\left(4 M_{4}^{2}-t\right)(-t)}{4}\left(z_{t}{ }^{2}-1\right)\right],
\end{aligned}
$$

where $E, E^{\prime}$ are the incident, final lab energies of the electron; $H_{+}, H_{-}$are defined in Sec. B 4 . It is conventional to express the cross section in terms of the Mott 
cross section:

$R_{\mathrm{He}}=\left(\frac{d \sigma}{d \Omega}\right) /\left(\frac{d \sigma}{d \Omega}\right)_{\mathrm{Mott}}$

$$
=\left[\left|\frac{t}{4 e^{2}} H_{-}\right|^{2}+\frac{4}{\left(4 M_{4}^{2}-t\right)\left(z_{t}^{2}-1\right)}\left|\frac{t}{4 e^{2}} H_{+}\right|^{2}\right]
$$

where

$$
\left(\frac{d \sigma}{d \Omega}\right)_{\mathrm{Mott}}=\left(\frac{2 e^{2}}{8 \pi E}\right)^{2} \frac{E^{\prime}}{E} \frac{\cos ^{2}(\theta / 2)}{\sin ^{2}(\theta / 2)} \text {. }
$$

We have used the relation

$$
z_{t}^{2}-1=\frac{\cot ^{2}(\theta / 2)}{1-t / 4 M_{4}^{2}}
$$

and $e^{2} / 4 \pi=1 / 137$. The electron mass has been consistently neglected in comparison with the momentum transfer. Using the large $z_{t}$ expressions for $H_{ \pm}$given in Sec. B 4, we see that $R_{\mathrm{He}}$ takes the form

$$
R_{\mathrm{He}}=z_{t}{ }^{2\left(\alpha_{1}-1\right)}\left[\mathfrak{S}_{0}(t)+t \mathfrak{S}_{1}(t) z_{t}^{\alpha_{1}-\alpha_{2}}+t^{2} \mathfrak{S}_{2}(t) z_{t}{ }^{2\left(\alpha_{2}-\alpha_{1}\right)}\right] .
$$

The term $\mathfrak{S}_{0}(t)$ in the limit of conventional electrodynamics (one-photon exchange) may be identified with the square of the helium form factor. The functions $\mathfrak{S}_{1}$ and $\mathfrak{S}_{2}$ measure the direct coupling of the electron to the scalar pion resonance. It would be interesting to see if such a coupling really existed. The functions $\mathfrak{S}_{0}, \mathfrak{S}_{1}$, and $\mathfrak{S}_{2}$ all have huge anomalous thresholds which reflect the size of the alpha particle and dominate the momentum-transfer dependence of $R_{\mathrm{He}}$ at any reasonable energy. In addition they reflect the isotopic scalar nucleon structure as is evident from Eqs. (4.10) and (4.11). Note finally that the S's are finite when $t=0$.
In order to study the dependence of $R_{\mathrm{He}}$ on $z_{t}$ experimentally, it is clear that measurements at several different energies, but the same momentum transfer, must be taken. Since $z_{t}$ is related to the energy by

$$
z_{t}=+\frac{M_{4} E+\frac{1}{4} t}{\left[\left(M_{4}^{2}-\frac{1}{4} t\right)\left(m^{2}-\frac{1}{4} t\right)\right]^{1 / 2}} \simeq \frac{2 E}{(-t)^{1 / 2}} \simeq \frac{1}{\sin (\theta / 2)},
$$

and we require a large $z_{t}$ for the asymptotic expansion to hold, the measurements must be made at forward angles. We expect that $\alpha_{1}(t)$ will be a monatonically decreasing function of momentum transfer $(-t)^{1 / 2}$, and hence to maximize the predicted energy dependence of $R_{\mathrm{He}}$, one should work at the largest possible values of $(-t)$. However, since $z_{t}$ must remain large, one is forced to large energies (and forward angles).

By way of illustration, if the $\mathfrak{S}_{1}$ and $\mathfrak{S}_{2}$ terms in $R_{\mathrm{He}}$ are ignored, one may write

$$
R_{\mathrm{He}}\left(t, E_{1}\right) / R_{\mathrm{He}}\left(t, E_{2}\right)=\left(z_{1} / z_{2}\right)^{2\left(\alpha_{1}-1\right)} \simeq e^{2\left(\alpha_{1}-1\right) \ln \left(E_{1} / E_{2}\right)} .
$$

If two measurements are made at $E_{1}=3 \mathrm{BeV}, E_{2}=6$ $\mathrm{BeV}$, and $t=-50 \mu^{2}$, then one finds $z_{1}=6\left(\theta_{1}=20^{\circ}\right)$, $Z_{2}=12\left(\theta_{2}=10^{\circ}\right)$, and

$$
R_{\mathrm{He}}\left(t, E_{1}\right) / R_{\mathrm{He}}\left(t, E_{2}\right)=\exp \left[1.4\left(1-\alpha_{1}\right)\right] .
$$

Defining the slope of $\alpha_{1}$ by the expansion

$$
\alpha_{1}(t)=1+t \alpha_{1}{ }^{\prime}+\ldots,
$$

we see that a $10 \%$ measurement of the ratio of the $R$ 's will detect an $\alpha_{1}{ }^{\prime}$ of $\left(1 / 700 \mu^{2}\right){ }^{16}$ This is to be compared with the slope of the nucleon trajectory which is about $\left(1 / 50 \mu^{2}\right)$.

We turn now to the case of electron-proton scattering which is considerably more complicated. In the laboratory system the cross section in terms of the Mott cross section is

$$
\begin{aligned}
& R_{N}=\left(\frac{d \sigma}{d \Omega}\right) /\left(\frac{d \sigma}{d \Omega}\right)_{\text {Mott }}=\left(\frac{t}{e^{2}}\right)^{2}\left\{\left(\frac{-t}{4 M^{2}}\right)\left(\frac{2}{x}-\frac{M^{2}}{P^{2}}\right)\left[\widetilde{G}_{1}^{2}-\frac{t P^{2}}{4} \widetilde{G}_{4}{ }^{2}+\frac{M^{2}}{2} \widetilde{G}_{3}^{2}-\frac{1}{2 t P^{2} x} G_{5}^{2}+\frac{1}{2} G_{6}{ }^{2}\right]\right. \\
& \left.-\frac{M^{2}}{P^{2}}\left[\widetilde{G}_{2}{ }^{2}+\frac{t}{8}\left(1+\frac{2 P^{2}}{M^{2}}\right) \widetilde{G}_{3}{ }^{2}-\frac{P^{2} t^{2}}{8 M^{2}} \widetilde{G}_{4}{ }^{2}+\frac{1}{4 M^{2}}\left(\frac{1}{M^{2}}-\frac{1}{2 P^{2}}\right) \frac{G_{5}{ }^{2}}{x}+\frac{t}{8 M^{2}} G_{6}{ }^{2}\right]-\frac{1}{4 M^{2} P^{2}} \frac{G_{5}{ }^{2}}{(1+x) x^{2}}-\frac{t^{2} P^{2}}{4 M^{2} x} \frac{\widetilde{G}_{4}{ }^{2}}{\left(2-M^{2} x / P^{2}\right)}\right\}, \\
& x=\cot ^{2}(\theta / 2)=\left(P^{2} / M^{2}\right)\left(1-z_{t}^{2}\right), \quad P^{2}=\frac{1}{4} t-M^{2}, \\
& \widetilde{G}_{3}=G_{3}-\frac{z_{t}\left(t P^{2}\right)^{1 / 2}}{t\left[M^{2}+P^{2}\left(1-z_{t}^{2}\right)\right]}\left[\frac{m t\left(1-z_{t}^{2}\right)}{1+z_{t}^{2}} G_{4}+2 M G_{5}\right] \\
& \widetilde{G}_{4}=G_{4}-\frac{8 M m z_{t}^{2} G_{5}}{\left(1-z_{t}^{2}\right)\left[M^{2}+P^{2}\left(1-z_{t}^{2}\right)\right]} \text {. } \\
& \text { nucleon problem by: }
\end{aligned}
$$$$
\begin{aligned}
& \widetilde{G}_{1}=G_{1}+\frac{1-z_{t}^{2}}{1+z_{t}^{2}}\left(m G_{3}+\frac{\left(t P^{2}\right)^{1 / 2}}{2} z_{t} G_{4}\right), \\
& \widetilde{G}_{2}=G_{1}+\frac{P^{2}}{M} G_{2}+\frac{m z_{t}^{2}}{1-z_{t}{ }^{2}} G_{3}-\frac{\left(t P^{2}\right)^{1 / 2}}{2} z_{t} G_{4}
\end{aligned}
$$$$
-\frac{2 m G_{5} P^{2} z_{t}}{M\left(t P^{2}\right)^{1 / 2}\left(1-z_{t}^{2}\right)}
$$

We have broken up the terms in a rather strange way for later convenience.

The ordinary analysis in terms of single photon

${ }^{16}$ A precision experiment on electron-helium scattering has been carried out by G. Burleson and H. Kendall, Nuclear Phys. 19, 68 (1960). Unfortunately, their energy was so low that the $z_{t}$ values were too small $(\sim 2)$ to trust the theory. 
exchange is obtained by setting $G_{3}=G_{4}=G_{5}=G_{6}=0$ and regarding $G_{1}$ and $G_{2}$ as functions of $t$ alone. In particular, if we make the identification

$$
\begin{aligned}
& \left(t / e^{2}\right) \widetilde{G}_{1}=F_{1}+2 M F_{2}, \\
& \left(t / e^{2}\right) \widetilde{G}_{2}=F_{1}+(t / 2 M) F_{2}
\end{aligned}
$$

(where $F_{1}$ and $F_{2}$ are the usual charge and magnetization form factors), we find the Rosenbluth formula in the form given by Hand, Miller, and Wilson. ${ }^{17}$ As in the electron-helium case, we find a modified form factor representation in the large $z_{t}$ regime. (Note that large $z_{t}$ implies large $x$.) To see how this comes about, we refer to Eqs. (2.12) which enable us to see the $z_{t}$ dependence of the individual $G$ 's [looking at a given angular momentum, e.g., Eq. (2.21)].

We remark that $G_{6}$ which did not contain the twopion state, would be expected to get its largest contribution from the exchange of a state with the quantum numbers of the $\pi^{0}$ meson and/or a pseudovector meson as we have discussed in Sec. B 2.

Using the large $z_{t}$ expressions for the $G$ 's given in Secs. B 2, and B 3 we see that the last two terms in $R_{N}$ are smaller than the leading terms by a factor of $z_{t}^{-4}$; neglecting these we find that $R_{N}$ may be written as

where

$$
R_{N}=z_{t}^{2\left(\alpha_{1}-1\right)}\left\{\left(\frac{-t}{4 M^{2}}\right)\left(\frac{2}{x}-\frac{M^{2}}{P^{2}}\right) \Re^{2}-\frac{M^{2}}{P^{2}} \mathcal{E}^{2}\right\},
$$

$$
\begin{aligned}
\mathscr{T}^{2}=\mathcal{G}_{0}(t)+\mathcal{G}_{1}(t) z_{t}^{\alpha_{2}-\alpha_{1}} & +\mathcal{G}_{2}(t) z_{t}^{\alpha_{3}-\alpha_{1}}+\mathcal{G}_{3}(t) z_{t}{ }^{2\left(\alpha_{2}-\alpha_{1}\right)} \\
& +\mathcal{G}_{4}(t) z_{t}{ }^{\left.2 \alpha_{3}-\alpha_{1}\right)}+\mathcal{G}_{5}(t) z_{t}{ }^{\alpha_{2}+\alpha_{3}-2 \alpha_{1}}
\end{aligned}
$$

and $\mathcal{E}^{2}$ has the same form with an independent set of $G$ 's. We have included here only a single trajectory, aside from the photon, in each isotopic spin state. The "pseudovector" negative $G$ parity contributions could be easily included. Although this structure is quite complicated, it should be possible to study the gross energy dependence. For sufficiently large $z_{t}$ the $\mathcal{G}_{0}$ term should be the most important, since we expect $\alpha_{2}-\alpha_{1}$ and $\alpha_{3}-\alpha_{1}$ to be negative for all $(-t)$ of interest. The same admonitions about the relative size of the energy, momentum transfer $(-\mathrm{t})$, and the scattering angle, made in connection with the helium scattering apply here.

Finally, it is clear that the foregoing analysis can be directly applied to electron-electron scattering. In this process we expect that the dominate contribution will come from $G_{1}$ alone, and hence would provide the cleanest experimental test of the Regge hypothesis for the photon. Of course, one must symmetrize the matrix element in this case, and this can easily be done.

\section{Discussion}

An experimental verification of the typical Regge energy dependence in high-energy electron scattering

${ }^{17}$ L. Hand, D. Miller and R. Wilson, Phys. Rev. Letters 8, 110 (1962). would lend considerable support to the idea that the photon is not an elementary particle in the usual sense. We cannot, however, be certain that conventional electrodynamics if accurately evaluated would not, through the accumulated effects of very high order multiple photon exchanges, lead to precisely the conjectured Regge behavior. After all, the Regge poles appear in potential scattering in just this way. It is, however, generally assumed that a field theory, involving the exchange of an elementary particle of spin $J$, leads to an asymptotic behavior of the form $z_{t}{ }^{J}$. This would have the consequence that at sufficiently high momentum transfers (where the "strongly" interacting Regge trajectories were suppressed) and at extreme energies $(>300-400 \mathrm{BeV})$ all charged particle cross sections would be dramatically dominated by the "weak" electromagnetic interactions. We find this a little difficult to accept. The distinction between weak and strong interactions seems hard to draw; if massive charged particles are Regge trajectories and this is a consequence of unitarity and analyticity of the $S$ matrix, there appears to be no reason why the photon should not share the property. It should be possible to prove that unless all particles are Regge trajectories, none are.

The question of what the nonelementary photon should be composed of is certainly a vexing one, as has been emphasized by Gell-Mann, et al. ${ }^{7}$ If it consists of mesons or nucleons, the profound violation of isotopic spin conservation in electromagnetic interactions is very mysterious; on the other hand, what binds leptons to yield such a state? We have no answer to these objections, but the way in which any of the conservation laws (e.g., baryon number) fit into a dynamical $S$ matrix approach is so completely obscure at present that such apparent difficulties may just be reflecting this ignorance. It also seems ridiculous to believe that the photon will behave like an elementary particle at ultra-high energies.

There are a host of problems raised by attempting to incorporate electrodynamics into an $S$-matrix formalism which makes no reference to the classical field concept. In an $S$-matrix theory there is no local current conservation although one would presumably impose the restriction that matrix elements between states of different charge vanish. One could speak of gauge transformations of the first kind only on states. It is quite unclear whether the universality of the residue of the photon Regge pole at $t=0$ (i.e., equality of charges) would emerge in any simple way. The problem of the approach to the classical limit is very troublesome also. $S$-matrix theory seems singularly unsuited to handle situations in which for a given total energy one may have an infinite number of particles. Of course one would like to recover also from the $S$-matrix electrodynamics the impressive agreement with experiments like the Lamb shift. In principle, the calculation of the Lamb shift would require a knowledge of the analytic structure of the $S$-matrix element $\gamma+e+p \rightarrow \gamma+e+p$, where $e$ 
and $p$ are in the hydrogen ground state, a rather formidable requirement. This being an essentially lowenergy phenomenon, we would expect any deviations of the photon spin from unity to have a very small effect, even if the slope of the photon trajectory were as large as that of the vacuum Regge pole.

In conclusion, we would like to draw attention to a few points which naturally arise. For example, the electron should be a Regge pole; this could be tested, in principle, by high-energy Compton scattering or by large-angle pair production. The photon trajectory is of course in reality somewhat schizoid, consisting in some sense of an isotopic scalar and isotopic vector part, although of course $\alpha_{1 V}(0)=\alpha_{1 S}(0)=1$. It is possible that the $\rho^{0}$ and $\omega$ mesons are "consorts" of the photon. Such a possibility would make the phenomenological model of Gell-Mann and Zachariasen ${ }^{18}$ (which couples these particles to the conventional electric current density) quite reasonable. Is there an even-signature "photon" family leading to bound states and resonances with even angular momentum? For example, could this be the socalled vacuum trajectory which would then be expected to be an isotopic schizoid also? If high-energy cross sections for particles and antiparticles should approach different constants, such a possibility would be suggestive.

It is hard to believe that we can ever acquire a deep understanding of the dynamical nature of isotopic spin unless we broaden the scope of the $S$-matrix approach to include electromagnetism.

${ }^{18}$ M. Gell-Mann and F. Zachariasen, Phys. Rev. 124, 953 (1961).

\title{
Extension of the Foldy-Wouthuysen Transformation
}

\author{
E. I. Blount \\ Bell Telephone Laboratories, Murray Hill, New Jersey
}

(Received July 23, 1962)

\begin{abstract}
By use of Moyal's representation and a perturbation theory developed by the author, we remove the interband matrix elements of the Dirac Hamiltonian in the presence of external fields. The result is exact in terms of velocity, but appears as a series in the field strengths and their derivatives, which is evaluated to second order in the fields and first order in their first derivatives.
\end{abstract}

\section{INTRODUCTION}

$I^{\mathrm{N}}$ N 1950, Foldy and Wouthuysen ${ }^{1}$ showed how one could derive the Pauli spin Hamiltonian from the Dirac equation by a unitary transformation. That is, they removed the matrix elements connecting positive and negative energy states. In the absence of external fields, this is no more than a transformation to eigenstates and was done exactly. When external fields are present, the problem is more complicated and could be done only by a series of successive approximations, amounting to an expansion in powers of $1 / \mathrm{m}$. The procedure was carried to second order and yielded a nonrelativistic result.

The author has recently developed a procedure for the removal of interband matrix elements in solid-state problems, ${ }^{2}$ which can easily be specialized to deal with the Dirac Hamiltonian, if we consider the positive and negative energy states as each constituting a doubly degenerate band. The separation thus obtained has a much greater range of validity than that of $\mathrm{FW}$, being valid for all energies. The result is obtained as a series in the field strengths and their derivatives, which we carry out to second order in the field strengths and first

${ }^{1}$ L. Foldy and S. Wouthuysen, Phys. Rev. 78, 29 (1950).

2 E. I. Blount in Solid State Physics, edited by F. Seitz and D. Turnbull (Academic Press Inc., New York, 1962), Vol. 13 order in their first derivatives, thus obtaining all terms found by $\mathrm{FW}$ and a number of others.

Moyal has shown that the multiplication of $\mathrm{two}^{3}$ operator functions of $\mathbf{p}$ and $\mathbf{x}$ may be written in the following form. If $P=\mathfrak{T} \mathcal{O}$, then

$$
\begin{gathered}
\odot(\mathbf{p}, \mathbf{x})=\mathscr{N}(\mathbf{p}, \mathbf{x}) \Theta(\mathbf{p}, \mathbf{x})+\frac{i}{2}\left(\frac{\partial \mathscr{N}}{\partial x_{i}} \frac{\partial \mathcal{O}}{\partial p_{i}}-\frac{\partial \mathscr{N}}{\partial p_{i}} \frac{\partial \mathcal{O}}{\partial x_{i}}\right) \\
-\frac{1}{8}\left(\frac{\partial^{2} \mathscr{N}}{\partial x_{i} \partial x_{j}} \frac{\partial^{2} \mathcal{O}}{\partial p_{i} \partial p_{j}}-\frac{2 \partial^{2} \mathscr{N}}{\partial p_{i} \partial x_{j}} \frac{\partial^{2} \mathcal{\partial}}{\partial p_{j} \partial x_{i}}\right. \\
\left.+\frac{\partial^{2} \mathscr{T}}{\partial p_{i} \partial p_{j}} \frac{\partial^{2} \mathcal{O}}{\partial x_{i} \partial x_{j}}\right)+\cdots, \quad(\hbar=1)
\end{gathered}
$$

where the multiplication on the right ignores the noncommutativity of $\mathbf{p}$ and $\mathbf{x}$. In this equation $\mathfrak{N}, \mathcal{O}, \mathcal{P}$ are all numerical functions of $\mathbf{p}$ and $\mathbf{x}$; to obtain the quantum-mechanical analogs it is only necessary to perform a Fourier transformation given in detail in reference 2 or to write out the power series for say, $\mathscr{N}$, in powers of $\mathbf{p}$ and $\mathbf{x}$, being careful in each order to use all possible permutations of $\mathbf{p}$ 's and $\mathbf{x}$ 's which may then be treated as operators. In the application of (1) to the

\footnotetext{
${ }^{3}$ J. Moyal, Proc. Cambridge Phil. Soc. 45, 99 (1949).
} 